\title{
Haptotropic shifts and fluxionality of cyclopentadienyl in mixed-hapticity complexes: a DFT mechanistic study
}

\author{
Carlos C. Romão, ${ }^{a}$ Luis F. Veiros ${ }^{\mathrm{b}, *}$ \\ ${ }^{a}$ Instituto de Tecnologia Química e Biológica, UNL, Av. da República, EAN, 2780-157, Oeiras, \\ Portugal. ${ }^{b}$ Centro de Química Estrutural, Complexo I, Instituto Superior Técnico, Av. Rovisco Pais 1, \\ 1049-001 Lisbon, Portugal; Phone: +351-218 419 283, Fax: +351-218 464457. \\ *e-mail address: veiros@ist.utl.pt
}




\section{Complementary information on the mechanisms}

Table S1. Relevant structural parameters for processes 3 and 4.

\begin{tabular}{cccc}
\hline & A & TS4 & TS5 \\
\hline Mo-N $(\AA)$ & $1.741,1.732$ & $1.741,1.731$ & $1.724,1.722$ \\
$<\mathrm{Mo}-\mathrm{C}\left(\eta^{5}-\mathrm{Cp}\right)>(\AA)$ & 2.48 & 2.48 & - \\
$\mathrm{Mo}-\mathrm{C}\left(\eta^{x}-\mathrm{Cp}\right)(\AA)$ & $2.334(x=1)$ & $2.503,2.518(x=2)$ & $2.228(2 \times)(x=1)$ \\
$\mathrm{N}-\mathrm{Mo}-\mathrm{N}\left({ }^{\circ}\right)$ & 108 & 106 & 108 \\
$\mathrm{Cp}-\mathrm{Mo}-\mathrm{Cp}\left({ }^{\circ}\right)^{\mathrm{a}}$ & 111 & 114 & 142
\end{tabular}

${ }^{\text {a }}$ Defined as: $\mathbf{A}-$ The midpoint of the $\eta^{5}-\mathrm{Cp}$ ring, the metal, and the coordinating carbon atom of $\eta^{1}-\mathrm{Cp}$. TS4 - The midpoint of the $\eta^{5}-\mathrm{Cp}$ ring, the metal, and the midpoint of the coordinated $\mathrm{C}-\mathrm{C}$ bond of $\eta^{2}-\mathrm{Cp}$. TS5 - The two coordinated $\mathrm{C}(\mathrm{Cp})$ atoms and the metal.
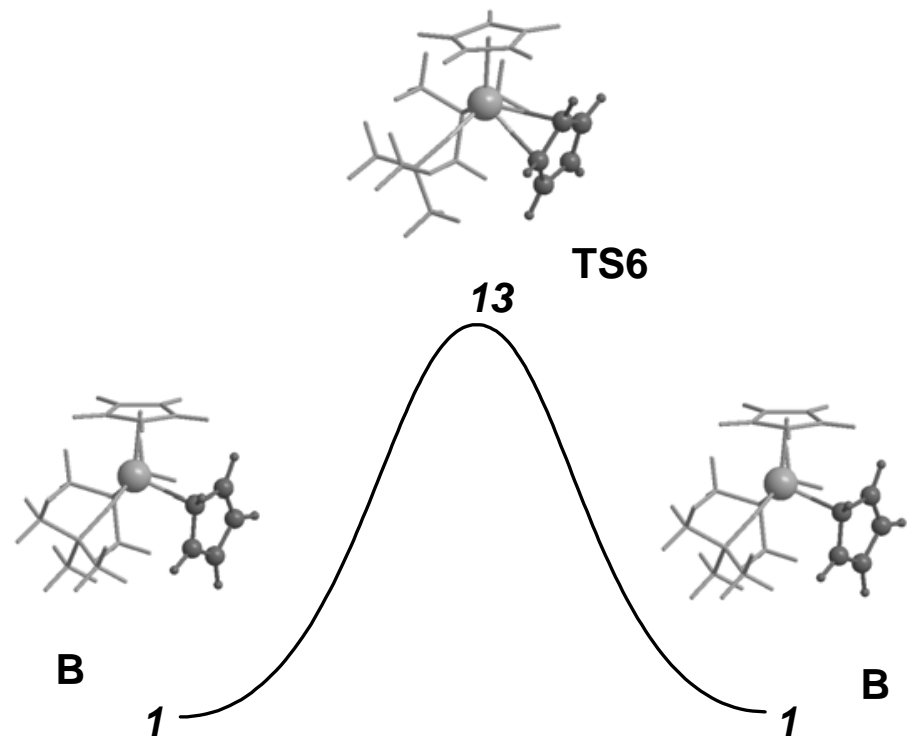

Figure S1. Energy profile $(\mathrm{PBE} 1 \mathrm{PBE} / \mathrm{b} 1)^{1}$ for the metallotropic migration along $\eta^{1}-\mathrm{Cp}$ in the less stable conformer $(\mathbf{B})$ of $\left[\mathrm{Mo}\left(\eta^{5}-\mathrm{Cp}\right)\left(\eta^{1}-\mathrm{Cp}\right)\left(\mathrm{N}^{t} \mathrm{Bu}\right)_{2}\right]$. The minimum and the transition state were optimized and the obtained structures are presented with $\eta^{1}-\mathrm{Cp}$ highlighted. The energy values (kcal $\mathrm{mol}^{-1}$, italics) are referred to $\mathbf{A}$, the most stable conformer.

\footnotetext{
${ }^{1}$ b1 stands for LanL2DZ plus f-polarization function on Mo, and a standard 6-31G(d,p) for the rest of the elements.
} 


\section{B3PW91; $R={ }^{t} \mathrm{Bu}$}

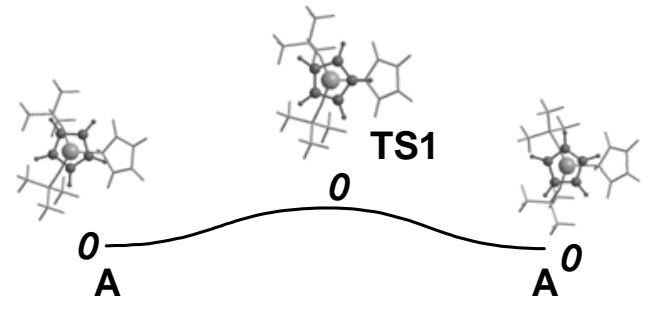

(1)

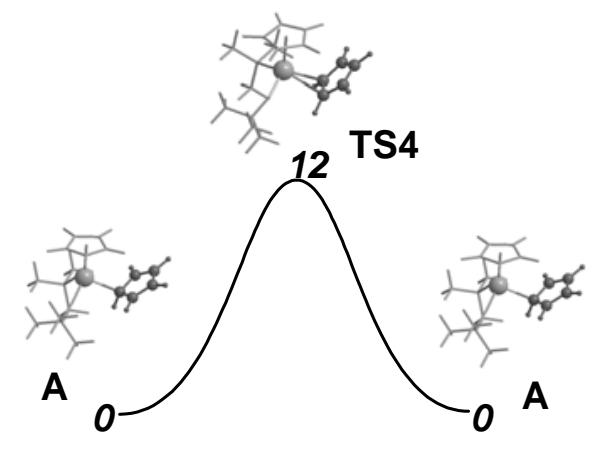

(3)

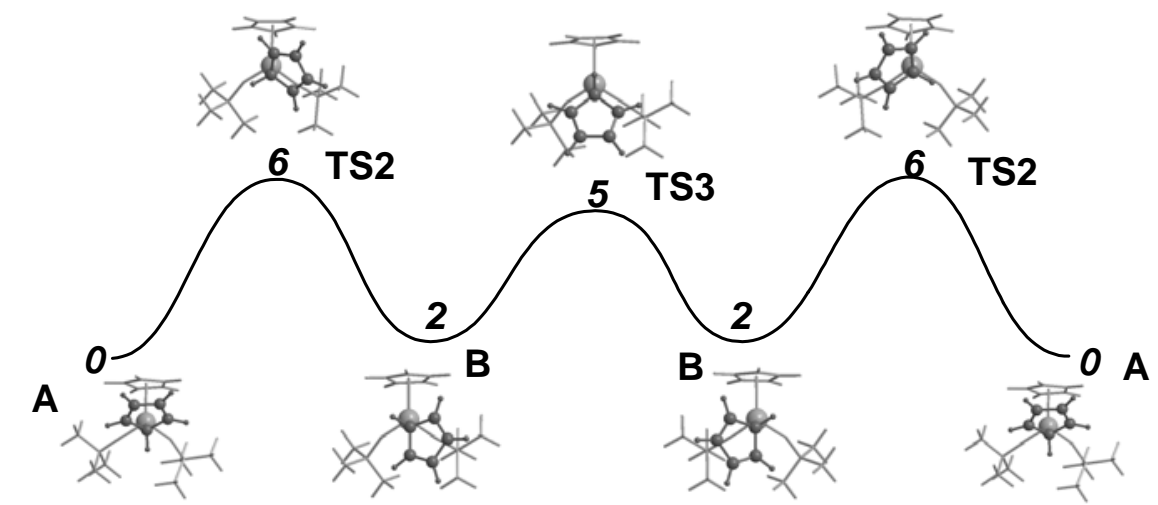

(2)

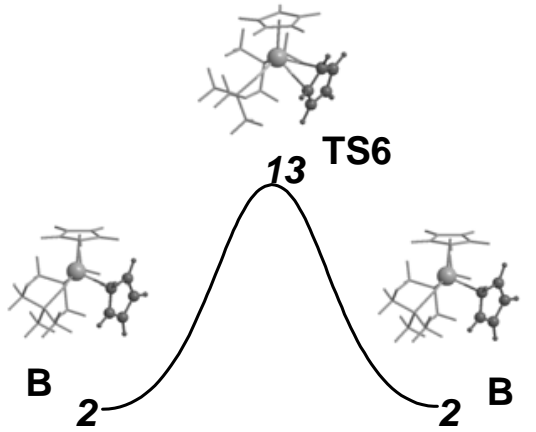

(4)

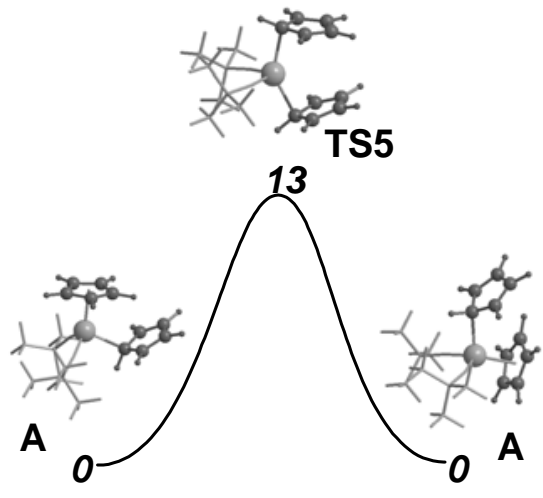

(5)

Figure S2. Energy profiles (B3PW91/b1) for all Cp fluxional processes in $\left[\mathrm{Mo}\left(\eta^{5}-\mathrm{Cp}\right)\left(\eta^{1}-\mathrm{Cp}\right)\left(\mathrm{N}^{t} \mathrm{Bu}\right)_{2}\right]$ : (1) rotation of $\eta^{5}-\mathrm{Cp}$, (2) rotation of $\eta^{1}-\mathrm{Cp}$, (3) metallotropic shift in $\mathbf{A}$, (4) metallotropic shift in $\mathbf{B}$, and (5) Cp exchange. The minima and the transition states were optimized and the obtained structures are presented with the moving $\mathrm{Cp}$ highlighted. The energy values $\left(\mathrm{kcal} \mathrm{mol}^{-1}\right.$, italics) are referred to the most stable conformer, $\mathbf{A}$. 


\section{B3LYP; $R={ }^{t} \mathrm{Bu}$}

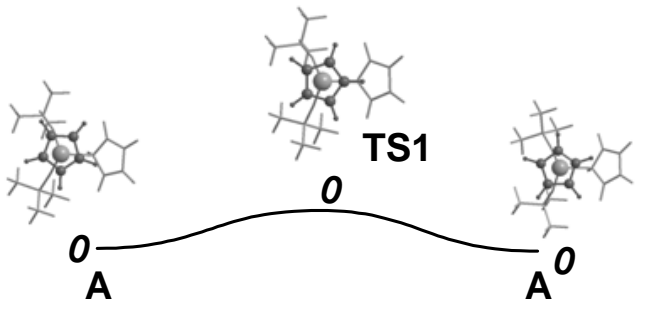

(1)

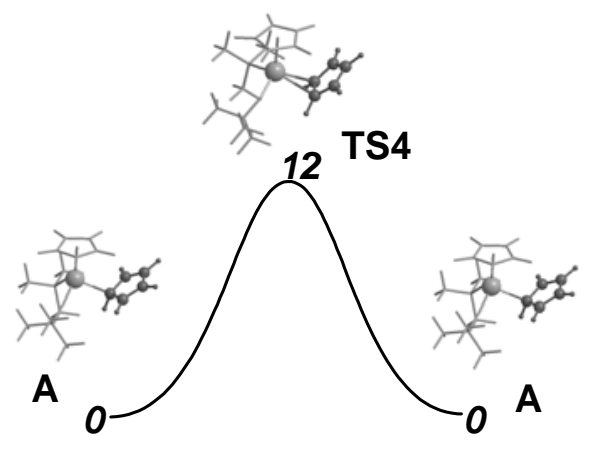

(3)

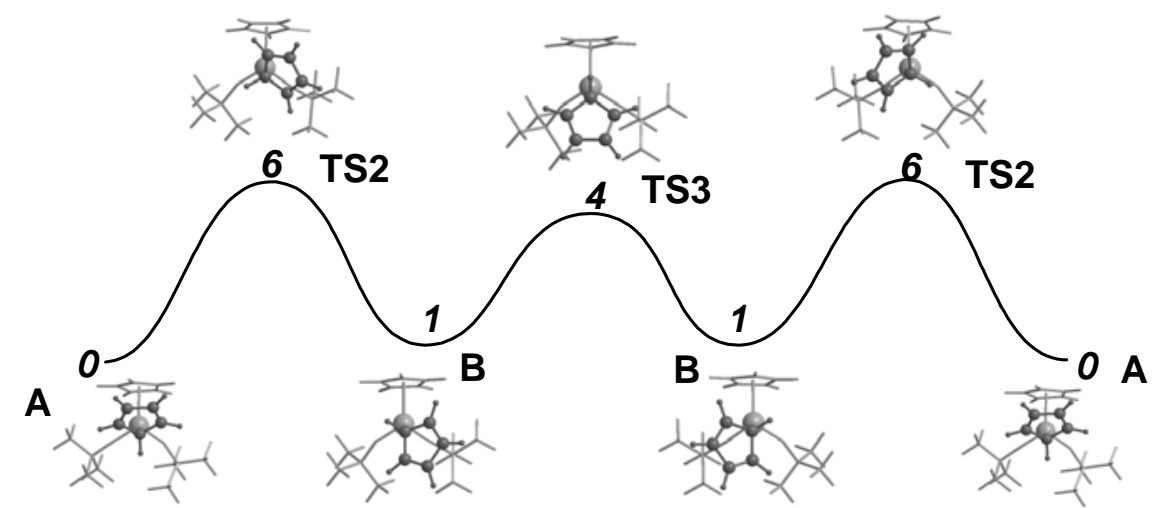

(2)

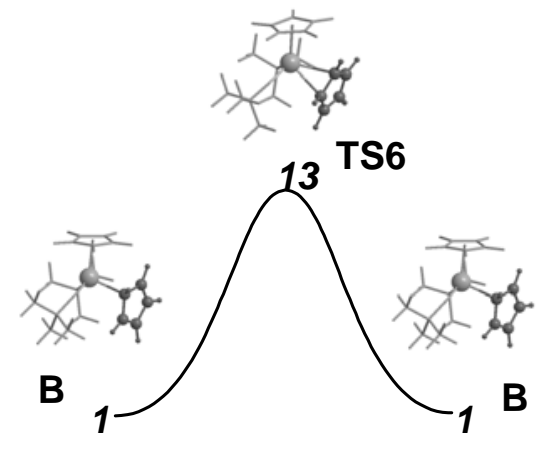

(4)

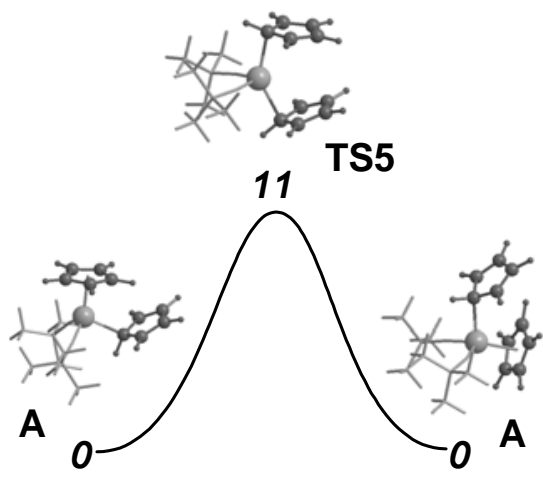

(5)

Figure S3. Energy profiles $(B 3 L Y P / b 1)$ for all $\mathrm{Cp}$ fluxional processes in $\left[\mathrm{Mo}\left(\eta^{5}-\mathrm{Cp}\right)\left(\eta^{1}-\mathrm{Cp}\right)\left(\mathrm{N}^{t} \mathrm{Bu}\right)_{2}\right]$ : (1) rotation of $\eta^{5}-\mathrm{Cp},(2)$ rotation of $\eta^{1}-\mathrm{Cp}$, (3) metallotropic shift in $\mathbf{A}$, (4) metallotropic shift in $\mathbf{B}$, and (5) Cp exchange. The minima and the transition states were optimized and the obtained structures are presented with the moving $\mathrm{Cp}$ highlighted. The energy values $\left(\mathrm{kcal} \mathrm{mol}^{-1}\right.$, italics) are referred to the most stable conformer, $\mathbf{A}$. 


\section{B3LYP; R = Me}

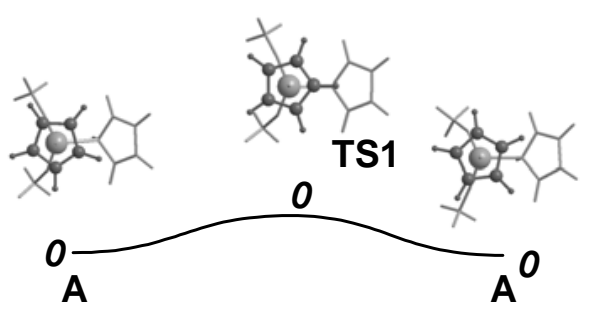

(1)

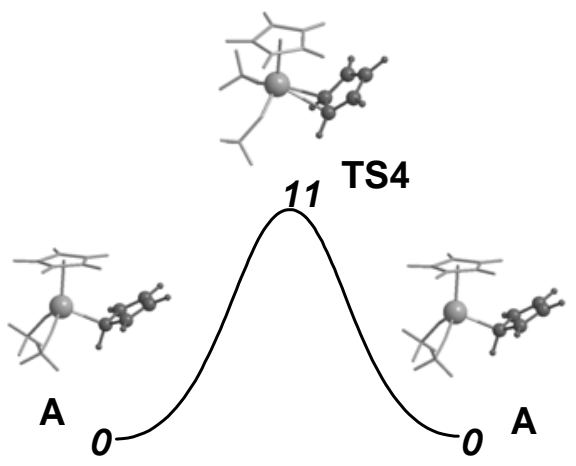

(3)

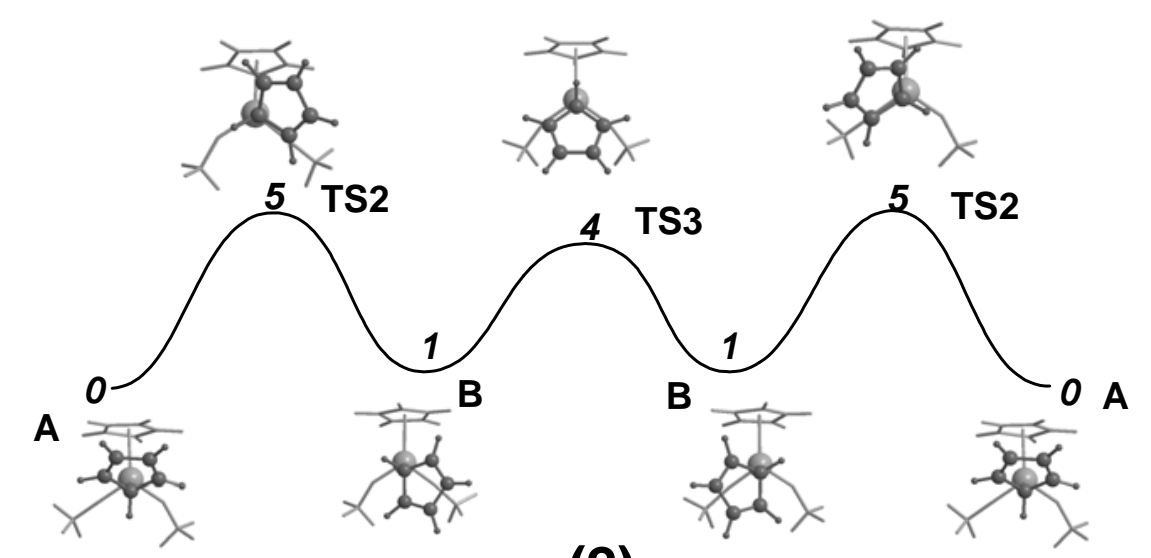

(2)

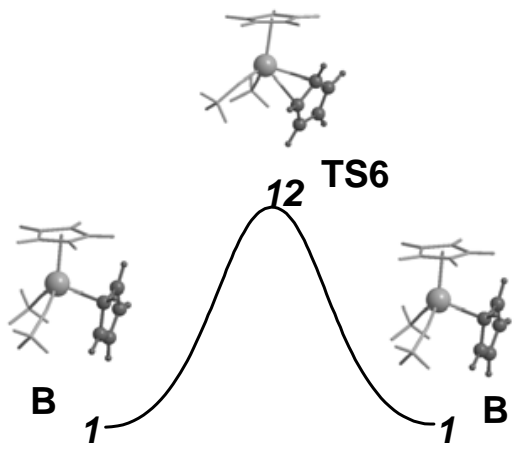

(4)

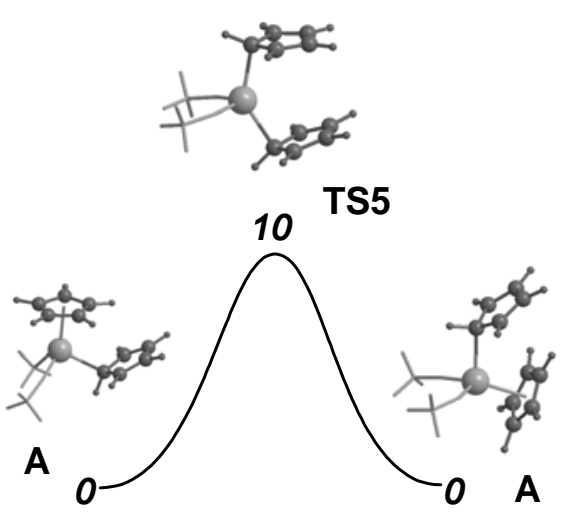

(5)

Figure S4. Energy profiles (B3LYP/b1) for all Cp fluxional processes in $\left[\mathrm{Mo}\left(\eta^{5}-\mathrm{Cp}\right)\left(\eta^{1}-\mathrm{Cp}\right)(\mathrm{NMe})_{2}\right]$ : (1) rotation of $\eta^{5}-\mathrm{Cp}$, (2) rotation of $\eta^{1}-\mathrm{Cp}$, (3) metallotropic shift in $\mathbf{A}$, (4) metallotropic shift in $\mathbf{B}$, and (5) $\mathrm{Cp}$ exchange. The minima and the transition states were optimized and the obtained structures are presented with the moving $\mathrm{Cp}$ highlighted. The energy values $\left(\mathrm{kcal} \mathrm{mol}^{-1}\right.$, italics) are referred to the most stable conformer, $\mathbf{A}$ 


\section{Atomic coordinates for all the optimized structures}

\section{PBE1PBE/b1; Mo $\left.\left(\eta^{5}-C p\right)\left(\eta^{1}-C p\right)\left(N^{t} B u\right)_{2}\right]$}

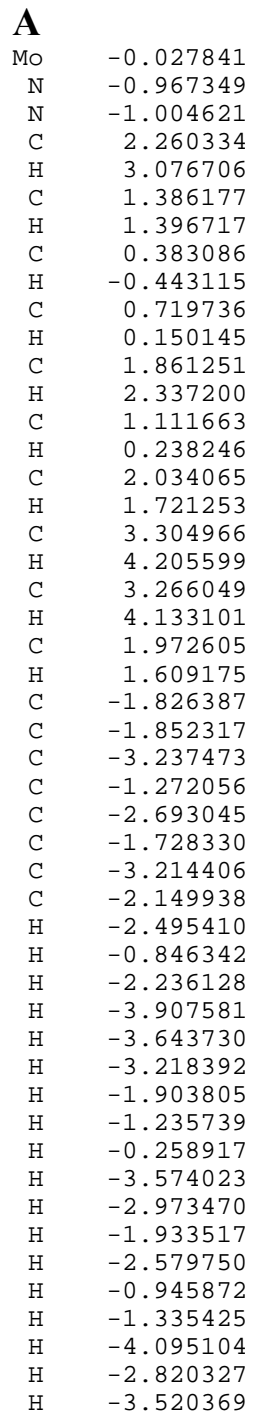

\begin{tabular}{|c|c|}
\hline ๑. 314479 & -0.660839 \\
\hline ๑.825233 & ๑.701693 \\
\hline$-\odot .768959$ & -1.611882 \\
\hline 1.349393 & -0.964875 \\
\hline ๑. 976408 & -0.362198 \\
\hline 2.395734 & -0.626015 \\
\hline 2.964317 & 0.293844 \\
\hline 2.462453 & -1.630596 \\
\hline 3.161256 & -1.663644 \\
\hline 1.518834 & -2.653049 \\
\hline 1.336883 & -3.554050 \\
\hline ๑. 821295 & -2.232497 \\
\hline$-\odot .004153$ & -2.743066 \\
\hline-1.266553 & ०. 409057 \\
\hline-1.838784 & ๑.739949 \\
\hline-1.971554 & -0.495879 \\
\hline-2.510690 & -1.382621 \\
\hline-1.832235 & -0.025460 \\
\hline-2.226073 & -0.484856 \\
\hline-1.076291 & 1.210226 \\
\hline$-\odot .822714$ & 1.811542 \\
\hline$-\odot .747402$ & 1.483684 \\
\hline - ๑ . 201994 & 2.346812 \\
\hline 1. 295491 & 1.749003 \\
\hline 0.225367 & 2.848771 \\
\hline 1.510587 & 1.188280 \\
\hline 2.613324 & 2.306044 \\
\hline-2.337015 & -0.864982 \\
\hline-2.459720 & -3.184926 \\
\hline-0.536439 & -2.543413 \\
\hline-1.522384 & -2.045770 \\
\hline$\odot .548282$ & 3.674201 \\
\hline ๑. 050666 & 3.240773 \\
\hline-0.720362 & 2.455549 \\
\hline 1.881760 & 1.971029 \\
\hline$\odot .572386$ & ๑.799955 \\
\hline 2.241647 & 0.374239 \\
\hline 2.986561 & 3.119223 \\
\hline 3.374975 & 1.521010 \\
\hline 2.466690 & 2.691850 \\
\hline$-2.91380 \odot$ & -1.166878 \\
\hline-1.674726 & -0.040792 \\
\hline-3.035643 & -0.500642 \\
\hline-3.053303 & -3.535357 \\
\hline-3.144761 & -2.845228 \\
\hline-1.883053 & -4.027483 \\
\hline-1.078723 & -2.903807 \\
\hline$\odot .068682$ & -3.365461 \\
\hline$\odot .134827$ & -1.736068 \\
\hline
\end{tabular}

B

$\begin{array}{rrrr}\text { Mo } & -0.666937 & -0.733288 & 0.669356 \\ \mathrm{~N} & -0.703729 & -1.608353 & -0.840622 \\ \mathrm{~N} & 0.524867 & 0.506632 & 0.550094 \\ \mathrm{C} & -2.992599 & -1.379003 & 1.333025 \\ \mathrm{H} & -3.356871 & -2.325424 & 0.957093 \\ \mathrm{C} & -2.998902 & -0.151100 & 0.632108 \\ \mathrm{H} & -3.430595 & 0.025487 & -0.344058 \\ \mathrm{C} & -2.376293 & 0.832239 & 1.465549 \\ \mathrm{H} & -2.238994 & 1.875348 & 1.213504 \\ \mathrm{C} & -1.976140 & 0.203558 & 2.659407 \\ \mathrm{H} & -1.426022 & 0.664389 & 3.468573 \\ \mathrm{C} & -2.323598 & -1.169471 & 2.560971 \\ \mathrm{H} & -2.120507 & -1.927858 & 3.306170 \\ \mathrm{C} & 0.482591 & -2.208929 & 1.857881 \\ \mathrm{H} & -0.240472 & -3.032690 & 1.891821 \\ \mathrm{C} & 1.751556 & -2.508946 & 1.169577 \\ \mathrm{H} & 1.814228 & -2.977291 & 0.195190 \\ \mathrm{C} & 2.789706 & -2.105519 & 1.951403 \\ \mathrm{H} & 3.843717 & -2.175908 & 1.703757 \\ \mathrm{C} & 2.259865 & -1.562570 & 3.183954 \\ \mathrm{H} & 2.858475 & -1.166465 & 3.997757 \\ \mathrm{C} & 0.901077 & -1.640963 & 3.153344 \\ \mathrm{H} & 0.225255 & -1.345601 & 3.946968 \\ \mathrm{C} & -0.383354 & -1.872900 & -2.217510\end{array}$

$\begin{array}{rrrr}\mathrm{C} & -0.195934 & -3.388329 & -2.374873 \\ \mathrm{C} & 0.890549 & -1.132915 & -2.635057 \\ \mathrm{C} & -1.570375 & -1.411017 & -3.074336 \\ \mathrm{C} & 2.786063 & 1.133419 & -0.018714 \\ \mathrm{C} & 1.576731 & 2.188533 & 1.920350 \\ \mathrm{C} & 0.846045 & 2.682629 & -0.439919 \\ \mathrm{C} & 1.428500 & 1.621799 & 0.501708 \\ \mathrm{H} & 0.636248 & -3.741975 & -1.759519 \\ \mathrm{H} & 0.018317 & -3.637537 & -3.419596 \\ \mathrm{H} & -1.100825 & -3.918749 & -2.065174 \\ \mathrm{H} & 1.133558 & -1.338110 & -3.683379 \\ \mathrm{H} & 1.734470 & -1.443955 & -2.012377 \\ \mathrm{H} & 0.759586 & -0.053452 & -2.513526 \\ \mathrm{H} & -1.383422 & -1.629516 & -4.131213 \\ \mathrm{H} & -1.724594 & -0.332925 & -2.966671 \\ \mathrm{H} & -2.486292 & -1.925391 & -2.768864 \\ \mathrm{H} & 3.506136 & 1.958841 & -0.021731 \\ \mathrm{H} & 2.691396 & 0.748774 & -1.037603 \\ \mathrm{H} & 3.166048 & 0.329951 & 0.617188 \\ \mathrm{H} & 2.289202 & 3.020568 & 1.930469 \\ \mathrm{H} & 1.931115 & 1.407223 & 2.598359 \\ \mathrm{H} & 0.612753 & 2.553941 & 2.288485 \\ \mathrm{H} & 1.515841 & 3.547555 & -0.488750 \\ \mathrm{H} & -0.131104 & 3.023281 & -0.083941 \\ \mathrm{H} & 0.725265 & 2.279384 & -1.449525\end{array}$

TS1

\begin{tabular}{|c|c|c|c|}
\hline Mo & -0.027060 & ๑. 339572 & -0.649703 \\
\hline $\mathrm{N}$ & - ๑. 975564 & 0.877630 & 0.698214 \\
\hline $\mathrm{N}$ & $-\odot .994056$ & -0.763251 & -1.585970 \\
\hline C & 2.295775 & 1.130966 & -1.175863 \\
\hline $\mathrm{H}$ & 3.126130 & 0.574287 & $-\odot .763771$ \\
\hline C & 1.646541 & 2.229191 & -0.560613 \\
\hline $\mathrm{H}$ & 1.886617 & 2.643028 & 0.409310 \\
\hline C & $\odot .550046$ & 2.589616 & -1.370618 \\
\hline $\mathrm{H}$ & -0.165131 & 3.376865 & -1.170958 \\
\hline C & ๑. 556871 & 1.742951 & -2.527193 \\
\hline $\mathrm{H}$ & -0.152534 & 1.783392 & -3.343080 \\
\hline C & 1.652662 & ๑. 863528 & -2.412406 \\
\hline $\mathrm{H}$ & 1.904450 & $\odot .063746$ & -3.095460 \\
\hline C & 1.085867 & -1.218688 & 0.474139 \\
\hline $\mathrm{H}$ & ๑. 203590 & -1.738631 & 0.863224 \\
\hline C & 1.960046 & -2.021739 & -0.396231 \\
\hline $\mathrm{H}$ & 1.607768 & -2.603979 & -1.239684 \\
\hline C & 3.243573 & -1.912195 & ०. . 046215 \\
\hline $\mathrm{H}$ & 4.116761 & -2.379412 & -0.397215 \\
\hline C & 3. 261868 & -1.067332 & 1.224547 \\
\hline $\mathrm{H}$ & 4.150898 & $-\odot .809276$ & 1.790665 \\
\hline C & 1.989787 & $-\odot .662686$ & 1.494409 \\
\hline $\mathrm{H}$ & 1.665603 & -0.040944 & 2.320764 \\
\hline C & -1.865812 & 1.327317 & 1.727519 \\
\hline C & -1.836700 & ๑. 284617 & 2.853588 \\
\hline C & -3.284524 & 1.448437 & 1.158179 \\
\hline C & -1.388278 & $2.68730 \odot$ & 2.253399 \\
\hline C & -2.627065 & -2.413883 & -0.911366 \\
\hline C & -1.631617 & -2.430642 & -3.221751 \\
\hline C & -3.198742 & -0.586316 & -2.544031 \\
\hline C & -2.102529 & -1.544467 & -2.061052 \\
\hline $\mathrm{H}$ & -2.500967 & ๑.590582 & 3.668677 \\
\hline $\mathrm{H}$ & -0.824346 & $\odot .176250$ & 3.253147 \\
\hline $\mathrm{H}$ & -2.165216 & $-\odot .690392$ & 2.482161 \\
\hline $\mathrm{H}$ & -3.978419 & 1.800204 & 1.929164 \\
\hline $\mathrm{H}$ & -3.634782 & $\odot .479235$ & $\odot .791974$ \\
\hline $\mathrm{H}$ & -3.303381 & 2.158907 & ๑. 326129 \\
\hline $\mathrm{H}$ & -2.042691 & 3.044824 & 3.055691 \\
\hline $\mathrm{H}$ & -1.391377 & 3.430439 & 1.450034 \\
\hline $\mathrm{H}$ & -0.369539 & 2.607820 & 2.643915 \\
\hline $\mathrm{H}$ & -3.478679 & -3.018116 & -1.242313 \\
\hline $\mathrm{H}$ & -2.946977 & -1.787053 & -0.074011 \\
\hline $\mathrm{H}$ & -1.843558 & -3.089560 & -0.554994 \\
\hline $\mathrm{H}$ & -2.456239 & -3.043056 & -3.602523 \\
\hline $\mathrm{H}$ & $-\odot .829837$ & -3.097706 & -2.891533 \\
\hline $\mathrm{H}$ & -1.248405 & -1.814826 & -4.040731 \\
\hline $\mathrm{H}$ & -4.053262 & -1.152312 & -2.930085 \\
\hline $\mathrm{H}$ & -2.819665 & ๑. 056107 & -3.344556 \\
\hline $\mathrm{H}$ & -3.540598 & ๑. 049851 & -1.722956 \\
\hline
\end{tabular}


TS2

$\begin{array}{rrrr}\text { Mo } & -0.115713 & 0.562338 & -0.228307 \\ \text { N } & -1.189808 & 1.395580 & 0.861355 \\ \text { N } & -0.957353 & -0.804961 & -0.868414 \\ \text { C } & 1.810064 & 2.127552 & -0.663552 \\ \text { H } & 2.411109 & 2.483919 & 0.162080 \\ \text { C } & 0.630951 & 2.717212 & -1.136979 \\ \text { H } & 0.133913 & 3.582549 & -0.721046 \\ \text { C } & 0.120528 & 1.887984 & -2.180539 \\ \text { H } & -0.774617 & 2.076341 & -2.759712 \\ \text { C } & 1.078736 & 0.856163 & -2.426561 \\ \text { H } & 0.978886 & 0.063548 & -3.155410 \\ \text { C } & 2.090697 & 0.974571 & -1.471461 \\ \text { H } & 2.916476 & 0.292881 & -1.322791 \\ \text { C } & 1.116405 & -0.476065 & 1.371569 \\ \text { H } & 0.662565 & 0.016581 & 2.236320 \\ \text { C } & 0.885666 & -1.933537 & 1.273428 \\ \text { H } & -0.090556 & -2.393413 & 1.363326 \\ \text { C } & 2.070951 & -2.563252 & 1.060089 \\ \text { H } & 2.216061 & -3.629180 & 0.919817 \\ \text { C } & 3.129855 & -1.573262 & 1.072202 \\ \text { H } & 4.186774 & -1.790671 & 0.956579 \\ \text { C } & 2.582664 & -0.346395 & 1.281933 \\ \text { H } & 3.124384 & 0.584669 & 1.399570 \\ \text { C } & -2.413256 & 1.725930 & 1.538338 \\ \text { C } & -2.097362 & 1.807384 & 3.038274 \\ \text { C } & -3.484715 & 0.663159 & 1.281248 \\ \text { C } & -2.886977 & 3.093897 & 1.029614 \\ \text { C } & -2.576242 & -2.600610 & -0.622231 \\ \text { C } & -0.574162 & -2.828223 & -2.127807 \\ \text { C } & -2.429042 & -1.229678 & -2.722313 \\ \text { C } & -1.629151 & -1.860443 & -1.575161 \\ \text { H } & -2.996561 & 2.080481 & 3.600511 \\ \text { H } & -1.324354 & 2.557762 & 3.226651 \\ \text { H } & -1.739567 & 0.841963 & 3.408533 \\ \text { H } & -4.419586 & 0.922247 & 1.790024 \\ \text { H } & -3.147697 & -0.311207 & 1.646217 \\ \text { H } & -3.684956 & 0.575484 & 0.208964 \\ \text { H } & -3.804883 & 3.395048 & 1.545494 \\ \text { H } & -3.092461 & 3.053182 & -0.044634 \\ \text { H } & -2.121768 & 3.855088 & 1.207876 \\ \text { H } & -3.098130 & -3.402258 & -1.155678 \\ \text { H } & -3.320921 & -1.916422 & -0.208028 \\ \text { H } & -2.018551 & -3.047528 & 0.205415 \\ \text { H } & -1.054877 & -3.675215 & -2.629235 \\ \text { H } & 0.055162 & -3.201878 & -1.315788 \\ \text { H } & 0.072038 & -2.319738 & -2.849329 \\ \text { H } & -1.763906 & -0.693494 & -3.405753 \\ \text { H } & -3.169604 & -0.523972 & -2.334319 \\ \text { H } & -2.951312 & -2.007914 & -3.288579\end{array}$

\section{TS3}

$\begin{array}{rrrr}\text { Mo } & -0.674450 & -0.653481 & 0.807370 \\ \mathrm{~N} & -0.524052 & -1.550845 & -0.671617 \\ \mathrm{~N} & 0.386449 & 0.706966 & 0.724551 \\ \mathrm{C} & -3.111279 & -1.118260 & 0.872861 \\ \mathrm{H} & -3.474962 & -1.744941 & 0.070404 \\ \mathrm{C} & -2.850340 & 0.282605 & 0.780885 \\ \mathrm{H} & -3.095111 & 0.919372 & -0.059742 \\ \mathrm{C} & -2.375709 & 0.721917 & 2.057014 \\ \mathrm{H} & -2.091043 & 1.734357 & 2.308538 \\ \mathrm{C} & -2.222153 & -0.404869 & 2.866380 \\ \mathrm{H} & -1.816152 & -0.419005 & 3.869676 \\ \mathrm{C} & -2.670746 & -1.551828 & 2.127447 \\ \mathrm{H} & -2.671206 & -2.573830 & 2.484127 \\ \mathrm{C} & 0.532065 & -1.980813 & 2.167704 \\ \mathrm{H} & -0.176204 & -2.195510 & 2.973032 \\ \mathrm{C} & 1.006524 & -3.171701 & 1.430683 \\ \mathrm{H} & 0.347195 & -3.920535 & 1.008555 \\ \mathrm{C} & 2.364173 & -3.153863 & 1.382220 \\ \mathrm{H} & 2.997569 & -3.883739 & 0.888859 \\ \mathrm{C} & 2.843677 & -1.996047 & 2.113040 \\ \mathrm{H} & 3.888312 & -1.734048 & 2.245749 \\ \mathrm{C} & 1.777003 & -1.311666 & 2.602189 \\ \mathrm{H} & 1.802386 & -0.420790 & 3.218875 \\ \mathrm{C} & -0.115351 & -1.874676 & -2.012224 \\ \mathrm{C} & -0.032455 & -3.401979 & -2.133709 \\ \mathrm{C} & 1.248713 & -1.248285 & -2.314783 \\ \mathrm{C} & -1.180673 & -1.329555 & -2.972536 \\ \mathrm{C} & 2.625031 & 1.450853 & 0.196437 \\ \mathrm{C} & 1.174209 & 2.723409 & 1.807138 \\ \mathrm{C} & 0.591315 & 2.683899 & -0.637855 \\ \mathrm{C} & 1.189844 & 1.881119 & 0.524260\end{array}$

$\begin{array}{rrrr}\mathrm{H} & 0.713164 & -3.794461 & -1.437662 \\ \mathrm{H} & 0.253861 & -3.686193 & -3.151890 \\ \mathrm{H} & -\Theta .999345 & -3.858092 & -1.901391 \\ \mathrm{H} & 1.579691 & -1.512917 & -3.325058 \\ \mathrm{H} & 1.992135 & -1.602311 & -1.594874 \\ \mathrm{H} & 1.190752 & -0.158386 & -2.243816 \\ \mathrm{H} & -\Theta .919558 & -1.579789 & -4.006283 \\ \mathrm{H} & -1.254187 & -0.241090 & -2.886462 \\ \mathrm{H} & -2.160200 & -1.762329 & -2.748225 \\ \mathrm{H} & 3.267101 & 2.332729 & 0.096842 \\ \mathrm{H} & 2.655542 & 0.888171 & -0.739629 \\ \mathrm{H} & 3.017904 & 0.808372 & 0.988195 \\ \mathrm{H} & 1.778962 & 3.627631 & 1.679939 \\ \mathrm{H} & 1.581110 & 2.149782 & 2.644639 \\ \mathrm{H} & 0.152834 & 3.024910 & 2.058795 \\ \mathrm{H} & 1.183361 & 3.587733 & -0.816274 \\ \mathrm{H} & -0.436818 & 2.982788 & -0.411297 \\ \mathrm{H} & 0.582712 & 2.086615 & -1.553976\end{array}$

\section{TS4}

\begin{tabular}{|c|c|c|c|}
\hline Mo & 0.164617 & ๑. 087454 & -0.489743 \\
\hline $\mathrm{N}$ & -0.513671 & ๑. 850561 & 0.920693 \\
\hline $\mathrm{N}$ & -1.136309 & -0.802097 & -1.205141 \\
\hline C & 2.319326 & 1.273078 & -1.088873 \\
\hline $\mathrm{H}$ & 3.161013 & 1.211452 & -0.414898 \\
\hline C & 1.277664 & 2.204871 & -1.038714 \\
\hline $\mathrm{H}$ & 1.148475 & 2.982301 & -0.298792 \\
\hline C & ๑. 321295 & 1.841119 & -2.037712 \\
\hline $\mathrm{H}$ & -0.592994 & 2.374203 & -2.265293 \\
\hline C & $\odot .877183$ & $\odot .761426$ & -2.797189 \\
\hline $\mathrm{H}$ & ๑. 392532 & ๑. 257977 & -3.622466 \\
\hline C & 2.072244 & ๑. 380927 & -2.186144 \\
\hline $\mathrm{H}$ & 2.690216 & $-\odot .465 \odot 84$ & -2.448335 \\
\hline C & 1.158433 & -2.171794 & $\odot .0 \odot 6251$ \\
\hline $\mathrm{H}$ & 0.230312 & -2.727694 & -0.013637 \\
\hline C & 2.319826 & -2.496213 & -0.725838 \\
\hline $\mathrm{H}$ & 2.354057 & -3.145735 & -1.593077 \\
\hline C & 3.412193 & -1.846791 & -0.130392 \\
\hline $\mathrm{H}$ & 4.439510 & -1.898051 & -0.476419 \\
\hline C & 2.958657 & -1.095533 & 0.960873 \\
\hline $\mathrm{H}$ & 3.565887 & -0.479318 & 1.614097 \\
\hline C & 1.562081 & -1.276749 & 1.076394 \\
\hline $\mathrm{H}$ & $\odot .980655$ & -1.078098 & 1.966636 \\
\hline C & -1.544323 & 1.260804 & 1.837029 \\
\hline C & -1.986684 & ๑. .035028 & 2.647372 \\
\hline C & -2.728430 & 1.820861 & 1.039514 \\
\hline C & -0.983595 & 2.337991 & 2.774607 \\
\hline C & -3.192211 & -2.037912 & -0.867634 \\
\hline C & -1.659659 & -2.542893 & -2.797654 \\
\hline C & -2.940101 & -0.378115 & -2.741205 \\
\hline C & -2.222114 & -1.436904 & -1.893556 \\
\hline $\mathrm{H}$ & -2.777264 & ๑. 311533 & 3.353240 \\
\hline $\mathrm{H}$ & -1.147365 & -0.373714 & 3.218064 \\
\hline $\mathrm{H}$ & -2.364899 & -0.746037 & 1.982314 \\
\hline $\mathrm{H}$ & -3.531698 & 2.132894 & 1.715541 \\
\hline $\mathrm{H}$ & -3.119389 & 1.062935 & ๑. 355325 \\
\hline $\mathrm{H}$ & -2.416463 & 2.688826 & 0.450181 \\
\hline $\mathrm{H}$ & -1.744102 & 2.661657 & 3.493565 \\
\hline $\mathrm{H}$ & $-\odot .656358$ & 3. 211228 & 2.202055 \\
\hline $\mathrm{H}$ & $-\odot .123261$ & 1.950823 & 3.328013 \\
\hline $\mathrm{H}$ & -4.021989 & -2.542523 & -1.374059 \\
\hline $\mathrm{H}$ & -3.604300 & -1.256269 & -0.223060 \\
\hline $\mathrm{H}$ & -2.678471 & -2.769138 & -0.236446 \\
\hline $\mathrm{H}$ & -2.470140 & -3.048124 & -3.333864 \\
\hline $\mathrm{H}$ & -1.119634 & -3.286720 & -2.205231 \\
\hline $\mathrm{H}$ & $-\odot .965703$ & -2.122248 & -3.530904 \\
\hline $\mathrm{H}$ & -3.778908 & -0.831671 & -3.279562 \\
\hline $\mathrm{H}$ & -2.255752 & $\odot .058466$ & -3.474501 \\
\hline $\mathrm{H}$ & $-3.3260 \odot 3$ & $\odot .425908$ & -2.107734 \\
\hline
\end{tabular}

\section{TS5}

$\begin{array}{rrrr}\text { Mo } & 0.271657 & -0.141382 & -0.552564 \\ \mathrm{~N} & -0.216508 & 0.773709 & 0.824189 \\ \mathrm{~N} & -1.136427 & -0.787779 & -1.303454 \\ \mathrm{C} & 2.117640 & 1.777339 & -1.338373 \\ \mathrm{H} & 2.275406 & 2.377670 & -0.450893 \\ \mathrm{C} & 0.823370 & 1.507844 & -1.946015 \\ \mathrm{H} & -0.011397 & 2.203768 & -1.883989 \\ \mathrm{C} & 1.095631 & 0.715395 & -3.134411 \\ \mathrm{H} & 0.342669 & 0.367239 & -3.830455 \\ \mathrm{C} & 2.443024 & 0.451593 & -3.175966 \\ \mathrm{H} & 2.950095 & -0.170817 & -3.904210 \\ \mathrm{C} & 3.074227 & 1.105898 & -2.063900\end{array}$




$\begin{array}{lrrr}\mathrm{H} & 4.128967 & 1.045499 & -1.821543 \\ \mathrm{C} & 1.080567 & -1.986347 & 0.398727 \\ \mathrm{H} & 0.225960 & -2.419907 & 0.915137 \\ \mathrm{C} & 1.627755 & -2.548195 & -0.826448 \\ \mathrm{H} & 1.060658 & -3.101193 & -1.565062 \\ \mathrm{C} & 2.961246 & -2.215974 & -0.887004 \\ \mathrm{H} & 3.631064 & -2.424240 & -1.713258 \\ \mathrm{C} & 3.318140 & -1.501788 & 0.307065 \\ \mathrm{H} & 4.299232 & -1.093727 & 0.521064 \\ \mathrm{C} & 2.203337 & -1.388482 & 1.102335 \\ \mathrm{H} & 2.139008 & -0.901127 & 2.067250 \\ \mathrm{C} & -1.004897 & 1.406896 & 1.849948 \\ \mathrm{C} & -0.047682 & 1.891114 & 2.947740 \\ \mathrm{C} & -1.999390 & 0.396988 & 2.432109 \\ \mathrm{C} & -1.742631 & 2.601466 & 1.234032 \\ \mathrm{C} & -2.457087 & -1.637514 & -3.124176 \\ \mathrm{C} & -3.424496 & 0.020445 & -1.505364 \\ \mathrm{C} & -2.905680 & -2.326953 & -0.748428 \\ \mathrm{C} & -2.476369 & -1.172377 & -1.661670 \\ \mathrm{H} & -0.605298 & 2.393000 & 3.745581 \\ \mathrm{H} & 0.681956 & 2.594313 & 2.536392 \\ \mathrm{H} & 0.496061 & 1.047343 & 3.382285 \\ \mathrm{H} & -2.585989 & 0.860877 & 3.231967 \\ \mathrm{H} & -1.469286 & -0.465308 & 2.847256 \\ \mathrm{H} & -2.687653 & 0.040388 & 1.661218 \\ \mathrm{H} & -2.330578 & 3.118928 & 1.999284 \\ \mathrm{H} & -2.417865 & 2.272311 & 0.439479 \\ \mathrm{H} & -1.029194 & 3.312428 & 0.807163 \\ \mathrm{H} & -3.454987 & -1.966640 & -3.432770 \\ \mathrm{H} & -1.759518 & -2.470067 & -3.251294 \\ \mathrm{H} & -2.142547 & -0.821919 & -3.781727 \\ \mathrm{H} & -4.436749 & -0.257519 & -1.817171 \\ \mathrm{H} & -3.090244 & 0.858753 & -2.123796 \\ \mathrm{H} & -3.464344 & 0.351908 & -0.464564 \\ \mathrm{H} & -3.913619 & -2.663743 & -1.012342 \\ \mathrm{H} & -2.906627 & -2.011008 & 0.298638 \\ \mathrm{H} & -2.219608 & -3.172608 & -0.852316\end{array}$

\section{TS6}

\begin{tabular}{|c|c|c|c|}
\hline Mo & $\odot .521179$ & 1.049958 & $-\odot .425249$ \\
\hline $\mathrm{N}$ & -0.929605 & 1.602399 & ๑. 350949 \\
\hline $\mathrm{N}$ & ๑. 385359 & -0.654352 & -0.609646 \\
\hline C & 1.622323 & 3.136524 & -1.622092 \\
\hline $\mathrm{H}$ & 2.070260 & 3.943362 & -1.055582 \\
\hline C & ๑. 275199 & 3. 028692 & -1.970089 \\
\hline $\mathrm{H}$ & -0.514052 & 3.713086 & -1.691468 \\
\hline C & ๑.091389 & 1.771879 & -2.624926 \\
\hline $\mathrm{H}$ & -0.831587 & 1.412040 & -3.060903 \\
\hline C & 1.386754 & 1.181224 & -2.794101 \\
\hline $\mathrm{H}$ & 1.586309 & $\odot .225460$ & -3.259172 \\
\hline C & 2.315091 & 1.992821 & -2.146921 \\
\hline $\mathrm{H}$ & 3.369210 & 1.781748 & -2.028081 \\
\hline C & 1.579296 & 1.200677 & 1.797159 \\
\hline $\mathrm{H}$ & 1.069929 & 2.074573 & 2.184624 \\
\hline C & 1.619443 & -0.069414 & 2.417676 \\
\hline $\mathrm{H}$ & ๑. 929137 & -0.417132 & 3.177422 \\
\hline C & 2.687603 & -0.794131 & 1.872123 \\
\hline $\mathrm{H}$ & 2.955139 & -1.812652 & 2.133816 \\
\hline C & 3. 319461 & -0.013363 & ๑. 895983 \\
\hline $\mathrm{H}$ & 4.163613 & -0.310195 & ๑.284547 \\
\hline C & 2.661680 & 1.237341 & $\odot .835443$ \\
\hline $\mathrm{H}$ & 3.091621 & 2.147651 & 0.438948 \\
\hline C & -2.227883 & 1.652591 & 0.961575 \\
\hline C & -2.105818 & 1.119110 & 2.395052 \\
\hline C & -3.207025 & ๑. 789344 & ๑.156827 \\
\hline C & -2.710150 & 3.109770 & $\odot .979609$ \\
\hline C & $-\odot .596749$ & -2.520288 & 0.581930 \\
\hline C & 1.284988 & -2.840379 & -1.057507 \\
\hline C & -0.994373 & -2.164586 & -1.883212 \\
\hline C & $\odot .017031$ & -2.036612 & -0.737480 \\
\hline $\mathrm{H}$ & -3.086500 & 1.119053 & 2.882659 \\
\hline $\mathrm{H}$ & -1.428186 & 1.744285 & 2.983404 \\
\hline $\mathrm{H}$ & -1.710892 & $\odot .099579$ & 2.389772 \\
\hline $\mathrm{H}$ & -4.212005 & 0.848562 & ๑.588346 \\
\hline $\mathrm{H}$ & -2.890252 & -0.256847 & ○.159398 \\
\hline $\mathrm{H}$ & -3.255203 & 1.132868 & -0.881141 \\
\hline $\mathrm{H}$ & -3.689704 & 3.187303 & 1.463550 \\
\hline $\mathrm{H}$ & -2.799737 & 3.497312 & -0.040018 \\
\hline $\mathrm{H}$ & -2.001323 & 3.738886 & 1.525662 \\
\hline $\mathrm{H}$ & $-\odot .845314$ & -3.584977 & ๑.516222 \\
\hline $\mathrm{H}$ & -1.510597 & -1.964646 & ๑.810233 \\
\hline $\mathrm{H}$ & $\odot .110095$ & -2.370245 & 1.401850 \\
\hline $\mathrm{H}$ & 1.049462 & -3.906809 & -1.140159 \\
\hline $\mathrm{H}$ & 2.028098 & -2.699567 & -0.268966 \\
\hline
\end{tabular}

$\begin{array}{lrrr}\mathrm{H} & 1.720811 & -2.504813 & -2.003197 \\ \mathrm{H} & -1.277091 & -3.214468 & -2.013456 \\ \mathrm{H} & -0.561900 & -1.805463 & -2.822029 \\ \mathrm{H} & -1.896831 & -1.583757 & -1.672783\end{array}$

\section{B3PW91/b1; Mo( $\left.\left(\eta^{5}-C p\right)\left(\eta^{1}-C p\right)\left(N^{t} B u\right)_{2}\right]$}

$\begin{array}{rrrr}\text { B } & & & \\ \text { Mo } & -0.641896 & -0.697220 & 0.677439 \\ \text { N } & -0.632612 & -1.544099 & -0.851445 \\ \text { N } & 0.533381 & 0.573587 & 0.618955 \\ \text { C } & -3.002352 & -1.406376 & 1.248780 \\ \text { H } & -3.348373 & -2.339660 & 0.825723 \\ \text { C } & -2.995637 & -0.150242 & 0.594897 \\ \text { H } & -3.403886 & 0.063554 & -0.383925 \\ \text { C } & -2.409963 & 0.806317 & 1.487309 \\ \text { H } & -2.279160 & 1.860482 & 1.283237 \\ \text { C } & -2.035633 & 0.131799 & 2.665293 \\ \text { H } & -1.519405 & 0.565328 & 3.510789 \\ \text { C } & -2.371193 & -1.241303 & 2.502870 \\ \text { H } & -2.186350 & -2.026347 & 3.224769 \\ \text { C } & 0.501412 & -2.198578 & 1.855004 \\ \text { H } & -0.209398 & -3.033227 & 1.824943 \\ \text { C } & 1.804826 & -2.459766 & 1.210355 \\ \text { H } & 1.916116 & -2.882405 & 0.219663 \\ \text { C } & 2.804814 & -2.089870 & 2.058053 \\ \text { H } & 3.869733 & -2.146373 & 1.856898 \\ \text { C } & 2.215808 & -1.607755 & 3.291528 \\ \text { H } & 2.775283 & -1.251378 & 4.150547 \\ \text { C } & 0.859127 & -1.689347 & 3.195432\end{array}$

\begin{tabular}{rr}
0.338997 & -0.619608 \\
0.913295 & 0.770971 \\
-0.750318 & -1.506242 \\
1.336391 & -1.049389 \\
0.993202 & -0.450623 \\
2.397315 & -0.737124 \\
3.007711 & 0.154816 \\
2.420526 & -1.724143 \\
3.123070 & -1.775141 \\
1.434717 & -2.714919 \\
1.216680 & -3.597369 \\
0.755359 & -2.287978 \\
-0.090111 & -2.773254 \\
-1.229665 & 0.458367 \\
-1.776411 & 0.840411 \\
-1.992051 & -0.441586 \\
-2.565924 & -1.293728 \\
-1.860892 & -0.009763 \\
-2.295138 & -0.473811 \\
-1.050527 & 1.193748 \\
-0.791179 & 1.760972 \\
-0.682408 & 1.487576 \\
-0.092528 & 2.335034 \\
1.328661 & 1.807646 \\
0.201834 & 2.853144 \\
1.571718 & 1.202271 \\
2.620243 & 2.455705 \\
-2.401428 & -0.920017 \\
-2.398951 & -3.189538 \\
-0.546793 & -2.558013 \\
-1.519508 & -2.036596 \\
0.483421 & 3.669853 \\
0.003887 & 3.274008 \\
-0.721516 & 2.398636 \\
1.901680 & 1.975737 \\
0.654214 & 0.751164 \\
2.343313 & 0.427719 \\
2.953011 & 3.258709 \\
3.421195 & 1.713245 \\
2.456539 & 2.878100 \\
-2.995983 & -1.297922 \\
-1.784447 & -0.090668 \\
-3.087230 & -0.535126 \\
-3.001155 & -3.610749 \\
-3.076178 & -2.835137 \\
-1.778757 & -3.986815 \\
-1.103362 & -2.983392 \\
0.099856 & -3.336870 \\
0.085824 & -1.747911 \\
- & \\
\hline
\end{tabular}
$-0.912152$

$-1.069520$

2.264036

3. 095838

$\odot .369997$

$-0.452142$

0.690867

0.105051

2. 307444

1.129543

$\odot .260894$

2. 015185

3. 301955

2.032335

1.703791

$-1.886552$

$-3.209800$

$-1.290008$

$-2.748077$

$-3.235875$

$-2.164780$

$-2.559950$

$-0.896724$

$-2.256766$

$-3.911946$

$-3.597363$

$-3.162448$

$-1.957014$

$-0.294250$

$-3.587113$

$-3.105170$

$-1.987351$

$-2.462968$

$-0.867703$

$-1.229810$

$-4.077994$

$-2.820794$

$-3.608663$

$-0.641896$

$-3.002352$

$-2.409963$

$-1.519405$

. 371193

0.501412

1.916116

$\odot .859127$

$-1.689347$ 


$\begin{array}{rrrr}\text { H } & 0.147803 & -1.437671 & 3.972736 \\ \mathrm{C} & -0.383143 & -1.870925 & -2.231192 \\ \mathrm{C} & -0.212493 & -3.397222 & -2.332864 \\ \mathrm{C} & 0.877704 & -1.160053 & -2.743133 \\ \mathrm{C} & -1.608560 & -1.433746 & -3.053262 \\ \mathrm{C} & 2.782292 & 1.151180 & -0.077360 \\ \mathrm{C} & 1.696118 & 2.246800 & 1.917793 \\ \mathrm{C} & 0.849163 & 2.739528 & -0.408769 \\ \mathrm{C} & 1.459967 & 1.669747 & 0.511010 \\ \mathrm{H} & 0.645649 & -3.732182 & -1.743310 \\ \mathrm{H} & -0.049831 & -3.691984 & -3.375207 \\ \mathrm{H} & -1.104677 & -3.908219 & -1.959745 \\ \mathrm{H} & 1.072953 & -1.419498 & -3.789732 \\ \mathrm{H} & 1.747693 & -1.446770 & -2.145777 \\ \mathrm{H} & 0.758322 & -0.074951 & -2.673697 \\ \mathrm{H} & -1.470784 & -1.694355 & -4.108330 \\ \mathrm{H} & -1.752891 & -0.351340 & -2.981948 \\ \mathrm{H} & -2.513098 & -1.929402 & -2.688576 \\ \mathrm{H} & 3.520751 & 1.959619 & -0.115572 \\ \mathrm{H} & 2.630362 & 0.770451 & -1.090627 \\ \mathrm{H} & 3.175731 & 0.338789 & 0.538601 \\ \mathrm{H} & 2.426542 & 3.062734 & 1.882224 \\ \mathrm{H} & 2.069762 & 1.465743 & 2.585203 \\ \mathrm{H} & 0.762072 & 2.636954 & 2.333737 \\ \mathrm{H} & 1.536520 & 3.586749 & -0.505606 \\ \mathrm{H} & -0.096585 & 3.109611 & -0.001152 \\ \mathrm{H} & 0.659615 & 2.330693 & -1.405458 \\ & & & \end{array}$

TS1

\begin{tabular}{|c|c|c|c|}
\hline Mo & -0.034451 & ๑. 349338 & -0.621961 \\
\hline $\mathrm{N}$ & $-\odot .945605$ & 0.925326 & 0.746174 \\
\hline $\mathrm{N}$ & -1.033071 & -0.765740 & -1.517121 \\
\hline C & 2.287075 & 1.115384 & -1.256317 \\
\hline $\mathrm{H}$ & 3.130558 & 0.564047 & -0.864536 \\
\hline C & 1.660757 & 2.225352 & -0.633396 \\
\hline $\mathrm{H}$ & 1.936915 & 2.654610 & 0.319949 \\
\hline C & 0.539977 & 2.581403 & -1.413888 \\
\hline $\mathrm{H}$ & -0.162629 & 3.377355 & -1.205032 \\
\hline c & 0.505346 & 1.716287 & -2.558418 \\
\hline $\mathrm{H}$ & $-\Theta .227436$ & 1.751890 & -3.353616 \\
\hline C & 1.603093 & 0.833982 & -2.468266 \\
\hline $\mathrm{H}$ & 1.830574 & 0.025543 & -3.149601 \\
\hline c & 1.114514 & -1.191762 & 0.506492 \\
\hline $\mathrm{H}$ & 0.241686 & -1.699311 & 0.931011 \\
\hline c & 1.967891 & -2.026665 & -0.359166 \\
\hline $\mathrm{H}$ & 1.596749 & -2.632579 & -1.177264 \\
\hline c & 3.261845 & -1.914441 & 0.055671 \\
\hline $\mathrm{H}$ & 4.122877 & -2.402364 & -0.389906 \\
\hline c & 3.309675 & -1.034461 & 1.209392 \\
\hline $\mathrm{H}$ & 4.211803 & -0.768225 & 1.750958 \\
\hline c & 2.044823 & $-\odot .612306$ & 1.493399 \\
\hline $\mathrm{H}$ & 1.741977 & 0.033746 & 2.308916 \\
\hline c & -1.865465 & 1.347602 & 1.767066 \\
\hline c & -1.907620 & $\odot .249276$ & 2.844088 \\
\hline $\mathrm{c}$ & -3.261229 & 1.536952 & 1.151860 \\
\hline c & -1.374428 & 2.670089 & 2. 381064 \\
\hline c & -2.679015 & -2.448703 & -0.935466 \\
\hline c & -1.571060 & -2.429589 & -3.200863 \\
\hline c & -3.197575 & -0.609780 & -2.581633 \\
\hline c & -2.108865 & -1.558497 & -2.052336 \\
\hline $\mathrm{H}$ & -2.588174 & 0.537974 & 3.652346 \\
\hline $\mathrm{H}$ & -0.913518 & 0.087531 & 3.270509 \\
\hline $\mathrm{H}$ & -2.255479 & -0.695477 & 2.416513 \\
\hline $\mathrm{H}$ & -3.974617 & 1.869047 & 1.914088 \\
\hline $\mathrm{H}$ & -3.622659 & 0.597576 & 0.724711 \\
\hline $\mathrm{H}$ & -3.231477 & 2.288451 & 0.356932 \\
\hline $\mathrm{H}$ & -2.052377 & 3. 005819 & 3.173609 \\
\hline $\mathrm{H}$ & -1.324972 & 3.452661 & 1.617799 \\
\hline $\mathrm{H}$ & -0.375629 & 2.544974 & 2.809202 \\
\hline $\mathrm{H}$ & -3.507039 & -3.058241 & -1.313718 \\
\hline $\mathrm{H}$ & -3.047400 & -1.836063 & -0.107816 \\
\hline $\mathrm{H}$ & -1.907224 & -3.120467 & -0.547926 \\
\hline $\mathrm{H}$ & -2.369194 & -3.049541 & -3.624240 \\
\hline $\mathrm{H}$ & -0.776247 & -3.089850 & -2.841719 \\
\hline $\mathrm{H}$ & -1.160255 & -1.802219 & -3.997538 \\
\hline $\mathrm{H}$ & -4.025145 & -1.185392 & -3.010219 \\
\hline H & -2.792363 & 0.043375 & -3.360286 \\
\hline & & & \\
\hline
\end{tabular}

\section{TS2}

\begin{tabular}{|c|c|c|c|}
\hline $\mathrm{N}$ & -1.236736 & 1.330569 & ๑.827292 \\
\hline $\mathrm{N}$ & -0.920220 & -0.854275 & -0.934825 \\
\hline C & 1.777817 & 2.178067 & -0.646369 \\
\hline $\mathrm{H}$ & 2.355635 & 2.546143 & ○. 190628 \\
\hline C & ๑. 588816 & 2.735999 & -1.134091 \\
\hline $\mathrm{H}$ & ๑. 063702 & 3.587781 & -0.725046 \\
\hline C & 0.117943 & 1.898969 & -2.193772 \\
\hline $\mathrm{H}$ & -0.768859 & 2.070671 & -2.790592 \\
\hline C & 1.109419 & ๑. 895587 & -2.430689 \\
\hline $\mathrm{H}$ & 1.044908 & 0.106088 & -3.166631 \\
\hline C & 2.101829 & 1.035078 & -1.455141 \\
\hline $\mathrm{H}$ & 2.946494 & ๑. 378177 & -1.301536 \\
\hline C & 1.109964 & $-\odot .489246$ & 1.358576 \\
\hline $\mathrm{H}$ & $\odot .623563$ & -0.015202 & 2.215690 \\
\hline C & $\odot .927455$ & -1.955823 & 1.257102 \\
\hline $\mathrm{H}$ & $-\odot .035435$ & -2.446715 & 1.321316 \\
\hline C & 2.138749 & -2.549225 & 1.080638 \\
\hline $\mathrm{H}$ & 2.321673 & -3.610741 & $\odot .949670$ \\
\hline C & 3.167120 & -1.525461 & 1.118894 \\
\hline $\mathrm{H}$ & 4.233359 & -1.711083 & 1.035348 \\
\hline C & 2.576114 & $-\odot .314007$ & 1.307571 \\
\hline $\mathrm{H}$ & 3.085528 & ๑. 633173 & 1.439490 \\
\hline C & -2.420630 & 1.722982 & 1.544934 \\
\hline C & -2.058396 & 1.763028 & 3.040445 \\
\hline C & -3.563577 & ๑. 726173 & 1.306439 \\
\hline C & -2.832077 & 3.127323 & 1.069969 \\
\hline C & -2.605116 & -2.591413 & -0.636622 \\
\hline C & $-\odot .611296$ & -2.926433 & -2.144106 \\
\hline C & -2.416693 & -1.279790 & -2.776806 \\
\hline C & -1.632876 & -1.906835 & -1.611218 \\
\hline $\mathrm{H}$ & -2.922824 & 2.085947 & 3.630495 \\
\hline $\mathrm{H}$ & -1.234042 & 2.459699 & 3.217806 \\
\hline $\mathrm{H}$ & -1.754745 & $\odot .771860$ & 3.390332 \\
\hline $\mathrm{H}$ & -4.463003 & 1.028670 & 1.854126 \\
\hline $\mathrm{H}$ & -3.273767 & -0.273341 & 1.642487 \\
\hline $\mathrm{H}$ & -3.809937 & 0.673613 & ๑.241757 \\
\hline $\mathrm{H}$ & -3.721083 & 3.467408 & 1.612053 \\
\hline $\mathrm{H}$ & -3.063990 & 3.119224 & ๑.000389 \\
\hline $\mathrm{H}$ & -2.024627 & 3.844509 & 1. 244514 \\
\hline $\mathrm{H}$ & -3.152184 & -3.392242 & -1.146132 \\
\hline $\mathrm{H}$ & -3.328632 & -1.872644 & -0.244324 \\
\hline $\mathrm{H}$ & -2.063368 & -3.030558 & ๑. 205615 \\
\hline $\mathrm{H}$ & -1.122780 & -3.763570 & -2.631855 \\
\hline $\mathrm{H}$ & ๑.000652 & -3.312012 & -1.324705 \\
\hline $\mathrm{H}$ & ๑. 056468 & -2.456381 & -2.871948 \\
\hline $\mathrm{H}$ & -1.737803 & -0.782647 & -3.475878 \\
\hline $\mathrm{H}$ & -3.134557 & -0.541545 & -2.407215 \\
\hline $\mathrm{H}$ & -2.964607 & -2.055845 & -3.321844 \\
\hline
\end{tabular}

\section{TS3}

$\begin{array}{rrrr}\text { Mo } & -0.658605 & -0.648681 & 0.807080 \\ \text { N } & -0.495961 & -1.530605 & -0.685219 \\ \text { N } & 0.392150 & 0.729303 & 0.748485 \\ \text { C } & -3.107154 & -1.142648 & 0.855576 \\ \text { H } & -3.463095 & -1.766458 & 0.047509 \\ \text { C } & -2.853016 & 0.262321 & 0.773596 \\ \text { H } & -3.097425 & 0.903114 & -0.064061 \\ \text { C } & -2.395570 & 0.697671 & 2.059142 \\ \text { H } & -2.123503 & 1.710855 & 2.320798 \\ \text { C } & -2.244224 & -0.434027 & 2.864713 \\ \text { H } & -1.852710 & -0.450844 & 3.873769 \\ \text { C } & -2.679423 & -1.580476 & 2.114082 \\ \text { H } & -2.680265 & -2.604190 & 2.465961 \\ \text { C } & 0.548077 & -1.985876 & 2.173831 \\ \text { H } & -0.177936 & -2.214098 & 2.959427 \\ \text { C } & 1.051758 & -3.171585 & 1.443128 \\ \text { H } & 0.410185 & -3.923470 & 0.999827 \\ \text { C } & 2.411627 & -3.148669 & 1.439658 \\ \text { H } & 3.064177 & -3.874630 & 0.965334 \\ \text { C } & 2.863450 & -1.991959 & 2.193045 \\ \text { H } & 3.902647 & -1.728967 & 2.363047 \\ \text { C } & 1.777906 & -1.312669 & 2.651129 \\ \text { H } & 1.780624 & -0.426788 & 3.275178 \\ \text { C } & -0.125314 & -1.874594 & -2.035239 \\ \text { C } & -0.093899 & -3.408116 & -2.152074 \\ \text { C } & 1.254961 & -1.292255 & -2.370379 \\ \text { C } & -1.193248 & -1.299481 & -2.981497 \\ \text { C } & 2.632531 & 1.468447 & 0.190071 \\ \text { C } & 1.197234 & 2.757356 & 1.810271 \\ \text { C } & 0.589011 & 2.702906 & -0.633876 \\ \text { C } & 1.197752 & 1.903400 & 0.530206 \\ \text { H } & 0.654252 & -3.823789 & -1.472493 \\ \text { H } & 0.159710 & -3.706027 & -3.175236 \\ \text { H } & -1.069018 & -3.833431 & -1.896466\end{array}$




$\begin{array}{rrrr}\mathrm{H} & 1.558749 & -1.576794 & -3.384087 \\ \mathrm{H} & 2.002086 & -1.661219 & -1.662377 \\ \mathrm{H} & 1.232483 & -0.200603 & -2.311347 \\ \mathrm{H} & -0.957093 & -1.560782 & -4.018716 \\ \mathrm{H} & -1.233956 & -0.209097 & -2.899705 \\ \mathrm{H} & -2.181765 & -1.701995 & -2.740919 \\ \mathrm{H} & 3.275805 & 2.348583 & 0.080690 \\ \mathrm{H} & 2.654439 & 0.902375 & -0.744172 \\ \mathrm{H} & 3.032904 & 0.829504 & 0.980803 \\ \mathrm{H} & 1.802040 & 3.659856 & 1.669769 \\ \mathrm{H} & 1.612586 & 2.190659 & 2.648257 \\ \mathrm{H} & 0.179542 & 3.063091 & 2.071445 \\ \mathrm{H} & 1.181670 & 3.604378 & -0.823296 \\ \mathrm{H} & -0.436070 & 3.007145 & -0.400693 \\ \mathrm{H} & \odot .570493 & 2.101255 & -1.546872\end{array}$

\section{TS4}

\begin{tabular}{|c|c|c|c|}
\hline Mo & $\odot .152416$ & $\odot .078479$ & -0.510281 \\
\hline $\mathrm{N}$ & -0.550775 & ๑. 833014 & 0.898127 \\
\hline $\mathrm{N}$ & -1.131719 & -0.825540 & -1.255374 \\
\hline C & 2.332711 & 1.278557 & -1.078592 \\
\hline $\mathrm{H}$ & $3.170 \odot 23$ & 1.211705 & $-\odot .399757$ \\
\hline C & 1.290085 & 2.210201 & -1.027116 \\
\hline $\mathrm{H}$ & 1.157946 & 2.983661 & -0.283691 \\
\hline C & ๑. 339497 & 1.855657 & -2.038156 \\
\hline $\mathrm{H}$ & $-\odot .569167$ & 2.395397 & -2.272273 \\
\hline C & 0.902175 & $\odot .782130$ & -2.804656 \\
\hline $\mathrm{H}$ & $\odot .425676$ & ๑. 289382 & -3.640766 \\
\hline C & 2.093009 & ๑. 394746 & -2.186610 \\
\hline $\mathrm{H}$ & 2.714906 & -0.446343 & -2.455306 \\
\hline C & 1.173997 & -2.183204 & -0.002112 \\
\hline $\mathrm{H}$ & $\odot .250646$ & -2.745204 & -0.044533 \\
\hline C & 2.356544 & -2.502002 & -0.704816 \\
\hline $\mathrm{H}$ & 2.417175 & -3.151031 & -1.571131 \\
\hline C & 3.431534 & -1.847613 & -0.079336 \\
\hline $\mathrm{H}$ & 4.468295 & -1.896095 & -0.397169 \\
\hline C & 2.946254 & -1.099188 & $1.0 \odot 2526$ \\
\hline $\mathrm{H}$ & 3.534551 & -0.483366 & 1.673444 \\
\hline C & 1.546400 & -1.286601 & 1.081010 \\
\hline $\mathrm{H}$ & $\odot .944010$ & -1.098488 & 1.959198 \\
\hline C & -1.550737 & 1.272430 & 1.838469 \\
\hline C & -1.995486 & $\odot .061361$ & 2.676712 \\
\hline C & -2.748495 & 1.847494 & 1. 065028 \\
\hline C & $-\odot .944975$ & 2.351655 & 2.752131 \\
\hline C & -3.173205 & -2.088960 & -0.878205 \\
\hline C & -1.692020 & -2.548645 & -2.865960 \\
\hline C & -2.989539 & -0.392018 & -2.733720 \\
\hline C & -2.235333 & -1.460101 & -1.922960 \\
\hline $\mathrm{H}$ & -2.767786 & ๑. 358926 & 3.394458 \\
\hline $\mathrm{H}$ & -1.151798 & -0.352730 & 3.236811 \\
\hline $\mathrm{H}$ & -2.398977 & -0.723636 & 2.031593 \\
\hline $\mathrm{H}$ & -3.528254 & 2.179979 & 1.758911 \\
\hline $\mathrm{H}$ & -3.171856 & 1. 091074 & $\odot .398898$ \\
\hline $\mathrm{H}$ & -2.438573 & 2.704496 & ๑. 458963 \\
\hline $\mathrm{H}$ & -1.680024 & 2.695071 & 3.488605 \\
\hline $\mathrm{H}$ & -0.619315 & 3.215007 & 2.163825 \\
\hline $\mathrm{H}$ & -0.076431 & 1.956323 & 3. 286814 \\
\hline $\mathrm{H}$ & -4.013724 & -2.590846 & -1.369982 \\
\hline $\mathrm{H}$ & -3.573263 & -1.323160 & -0.207753 \\
\hline $\mathrm{H}$ & -2.638067 & -2.828391 & -0.274956 \\
\hline $\mathrm{H}$ & -2.515050 & -3.051174 & -3.385794 \\
\hline $\mathrm{H}$ & -1.128318 & -3.299077 & -2.304921 \\
\hline $\mathrm{H}$ & -1.025043 & -2.110924 & -3.614150 \\
\hline $\mathrm{H}$ & -3.839649 & -0.844526 & -3.255445 \\
\hline $\mathrm{H}$ & -2.331238 & ๑. . 063018 & -3.479408 \\
\hline $\mathrm{H}$ & -3.364647 & $\odot .397994$ & $-2.0769 \odot \odot$ \\
\hline
\end{tabular}

\section{TS5}

$\begin{array}{rrrr}\text { Mo } & 0.242184 & -0.141672 & -0.547203 \\ \mathrm{~N} & -0.239892 & 0.778138 & 0.837335 \\ \mathrm{~N} & -1.162065 & -0.796518 & -1.315201 \\ \mathrm{C} & 2.113665 & 1.820580 & -1.326515 \\ \mathrm{H} & 2.231963 & 2.430801 & -0.439648 \\ \mathrm{C} & 0.835679 & 1.484534 & -1.946897 \\ \mathrm{H} & -0.013008 & 2.167480 & -1.923463 \\ \mathrm{C} & 1.164101 & 0.706184 & -3.137043 \\ \mathrm{H} & 0.438853 & 0.325168 & -3.845099 \\ \mathrm{C} & 2.522993 & 0.512088 & -3.163003 \\ \mathrm{H} & 3.071032 & -0.077205 & -3.889541 \\ \mathrm{C} & 3.110333 & 1.199538 & -2.041118 \\ \mathrm{H} & 4.166293 & 1.202067 & -1.795478 \\ \mathrm{C} & 1.093590 & -1.972018 & 0.388817 \\ \mathrm{H} & 0.253192 & -2.394550 & 0.938572\end{array}$

$\begin{array}{lrrr}\mathrm{C} & 1.614392 & -2.592526 & -0.824966 \\ \mathrm{H} & 1.023112 & -3.150407 & -1.540545 \\ \mathrm{C} & 2.957676 & -2.311659 & -0.905043 \\ \mathrm{H} & 3.616720 & -2.579106 & -1.723242 \\ \mathrm{C} & 3.350803 & -1.572175 & 0.267235 \\ \mathrm{H} & 4.350125 & -1.200568 & 0.463957 \\ \mathrm{C} & 2.248784 & -1.394142 & 1.067463 \\ \mathrm{H} & 2.212312 & -0.880518 & 2.020080 \\ \mathrm{C} & -1.016737 & 1.423850 & 1.869828 \\ \mathrm{C} & -0.041331 & 1.916789 & 2.953322 \\ \mathrm{C} & -2.008899 & 0.420793 & 2.477039 \\ \mathrm{C} & -1.762147 & 2.618415 & 1.253819 \\ \mathrm{C} & -2.483085 & -1.650042 & -3.141795 \\ \mathrm{C} & -3.461169 & 0.004547 & -1.517360 \\ \mathrm{C} & -2.933805 & -2.347174 & -0.762941 \\ \mathrm{C} & -2.504809 & -1.186232 & -1.674717 \\ \mathrm{H} & -0.587396 & 2.430201 & 3.752080 \\ \mathrm{H} & 0.686743 & 2.612729 & 2.527068 \\ \mathrm{H} & 0.503894 & 1.076227 & 3.392014 \\ \mathrm{H} & -2.584218 & 0.894869 & 3.279445 \\ \mathrm{H} & -1.477681 & -0.439166 & 2.895466 \\ \mathrm{H} & -2.708993 & 0.058402 & 1.719661 \\ \mathrm{H} & -2.339602 & 3.142509 & 2.022831 \\ \mathrm{H} & -2.448873 & 2.285651 & 0.470807 \\ \mathrm{H} & -1.054335 & 3.325994 & 0.812312 \\ \mathrm{H} & -3.479808 & -1.983407 & -3.450417 \\ \mathrm{H} & -1.782572 & -2.479713 & -3.271784 \\ \mathrm{H} & -2.173303 & -0.832572 & -3.799203 \\ \mathrm{H} & -4.473220 & -0.278901 & -1.825678 \\ \mathrm{H} & -3.134670 & 0.844447 & -2.137704 \\ \mathrm{H} & -3.500528 & 0.337106 & -0.476896 \\ \mathrm{H} & -3.940973 & -2.685607 & -1.028633 \\ \mathrm{H} & -2.937542 & -2.034823 & 0.285108 \\ \mathrm{H} & -2.246778 & -3.191905 & -0.867428\end{array}$

\section{TS6}

\begin{tabular}{|c|c|c|c|}
\hline Mo & ๑. 518865 & 1.027250 & -0.423148 \\
\hline $\mathrm{N}$ & -0.941082 & 1.560327 & ๑. 361032 \\
\hline $\mathrm{N}$ & 0.407885 & -0.684165 & -0.629242 \\
\hline C & 1.604711 & 3.164027 & -1.620577 \\
\hline $\mathrm{H}$ & 2.047224 & 3.974939 & -1.055463 \\
\hline C & ๑. 255115 & 3. 038532 & -1.955273 \\
\hline $\mathrm{H}$ & $-\odot .539624$ & 3.714384 & -1.672055 \\
\hline C & ๑. 081593 & 1.780600 & -2.616539 \\
\hline $\mathrm{H}$ & $-\odot .838212$ & 1.416128 & -3.055196 \\
\hline C & 1. 385123 & 1. 208471 & -2.800540 \\
\hline $\mathrm{H}$ & 1.594779 & $\odot .260862$ & -3.277321 \\
\hline C & 2.308513 & 2.030354 & -2.156161 \\
\hline $\mathrm{H}$ & 3.367232 & 1.835310 & -2.050845 \\
\hline C & 1.590882 & 1.179959 & 1.810714 \\
\hline $\mathrm{H}$ & 1.065460 & 2.041423 & 2.204009 \\
\hline C & 1.667259 & $-\odot .089913$ & 2.430853 \\
\hline $\mathrm{H}$ & $\odot .993471$ & -0.454784 & 3.197344 \\
\hline C & 2.750426 & $-\odot .788722$ & 1.876021 \\
\hline $\mathrm{H}$ & 3.047026 & -1.799320 & 2.137443 \\
\hline C & 3.356549 & ๑. 008832 & ๑.894989 \\
\hline $\mathrm{H}$ & 4.204892 & -0.266317 & 0.279054 \\
\hline C & 2.666187 & 1.244307 & ๑.839238 \\
\hline $\mathrm{H}$ & 3.075052 & 2.165713 & $\odot .446532$ \\
\hline C & -2.243122 & 1.656596 & 0.965937 \\
\hline C & -2.148246 & 1.135593 & 2.410078 \\
\hline C & -3.247621 & ๑. 813719 & 0.163422 \\
\hline C & -2.682909 & 3.131641 & $\odot .966641$ \\
\hline C & $-\odot .612877$ & -2.535887 & $\odot .570375$ \\
\hline C & 1.288679 & -2.890291 & -1.046883 \\
\hline C & $-\odot .978280$ & -2.197335 & -1.907001 \\
\hline C & $\odot .023268$ & -2.067213 & -0.747802 \\
\hline $\mathrm{H}$ & -3.132034 & 1.174095 & 2.890410 \\
\hline $\mathrm{H}$ & -1.455326 & 1.744934 & 2.996997 \\
\hline $\mathrm{H}$ & -1.788985 & ๑. 103312 & 2.421964 \\
\hline $\mathrm{H}$ & -4.251769 & ๑.906061 & ๑.591601 \\
\hline H & -2.963225 & $-\odot .241599$ & 0.175494 \\
\hline $\mathrm{H}$ & -3.284272 & 1.148936 & -0.877638 \\
\hline $\mathrm{H}$ & -3.660803 & 3.243165 & 1.447777 \\
\hline $\mathrm{H}$ & -2.761038 & 3.511706 & -0.056678 \\
\hline $\mathrm{H}$ & -1.958065 & 3.746911 & 1.507603 \\
\hline $\mathrm{H}$ & $-\odot .873092$ & -3.598326 & $\odot .509611$ \\
\hline $\mathrm{H}$ & -1.523097 & -1.969112 & 0.784235 \\
\hline $\mathrm{H}$ & ๑. 084955 & -2.389601 & 1. 398491 \\
\hline $\mathrm{H}$ & 1.041366 & -3.954641 & -1.124960 \\
\hline $\mathrm{H}$ & 2.023945 & -2.754852 & -0.250227 \\
\hline $\mathrm{H}$ & 1.741355 & -2.567868 & -1.989163 \\
\hline $\mathrm{H}$ & -1.272946 & -3.245269 & -2.027895 \\
\hline $\mathrm{H}$ & -0.531211 & -1.856037 & -2.845514 \\
\hline
\end{tabular}




\section{B3LYP/b1; Mo $\left.\left(\eta^{5}-C p\right)\left(\eta^{1}-C p\right)\left(N^{t} B u\right)_{2}\right]$}

$\begin{array}{rr}0.368541 & -\oplus .620284 \\ 0.956205 & 0.783589 \\ -0.707842 & -1.504097 \\ 1.413163 & -1.020024 \\ 1.121459 & -0.376218 \\ 2.457784 & -0.787962 \\ 3.107423 & 0.075751 \\ 2.410700 & -1.819889 \\ 3.095041 & -1.941192 \\ 1.400390 & -2.760679 \\ 1.131593 & -3.658016 \\ 0.767648 & -2.253655 \\ -0.080341 & -2.684056 \\ -1.242733 & 0.456974 \\ -1.772203 & 0.850243 \\ -2.035176 & -0.446118 \\ -2.604805 & -1.293218 \\ -1.934906 & -0.022489 \\ -2.394164 & -0.488047 \\ -1.116670 & 1.180520 \\ -0.879101 & 1.740186 \\ -0.715854 & 1.482344 \\ -0.114435 & 2.329296 \\ 1.330553 & 1.819882 \\ 0.203021 & 2.874685 \\ 1.504153 & 1.211239 \\ 2.653923 & 2.464493 \\ -2.398630 & -0.907649 \\ -2.351666 & -3.201594 \\ -0.528926 & -2.523469 \\ -1.490432 & -2.027706 \\ 0.456023 & 3.690159 \\ 0.055308 & 3.296704 \\ -0.739943 & 2.426379 \\ 1.803287 & 1.983405 \\ 0.567885 & 0.763871 \\ 2.274339 & 0.433942 \\ 2.958214 & 3.263256 \\ 3.453594 & 1.718068 \\ 2.540699 & 2.891364 \\ -3.007269 & -1.280393 \\ -1.799243 & -0.066496 \\ -3.071909 & -0.540736 \\ -2.962441 & -3.617539 \\ -3.019955 & -2.866007 \\ -1.716569 & -3.998390 \\ -1.097270 & -2.941189 \\ 0.133087 & -3.302761 \\ 0.088650 & -1.702078\end{array}$

$-1.702078$

0.674250 $-0.861519$

0.630653

1.262957

0.840606

0.609406

$-0.367835$

1.506072

1.303974

2.684785

3. 532967

2. 520908

3.242584

1.859670

1.832114

1.221234

0.230241

2. 075044

1.881097

3.311125

4.173491

3. 208445

3. 981271

$-2.243881$

$-2.331568$
0.887250

$-1.603468$

2.775240

1.746275

0.854630

1.469510

$\odot .675543$

$-0.011874$

$-1.071524$

1. 086911

1.759204

0.758416

$-1.458995$

$-1.759365$

$-2.504690$

3. 525536

2. 596951

3.170120

2. 488279

2.122810

0.828032

1.553276

$-0.076049$

$\odot .637240$

TS1

$\begin{array}{rr}\mathrm{Mo} & -0 . \\ \mathrm{N} & - \\ \mathrm{N} & -1 \\ \mathrm{C} & \\ \mathrm{H} & \end{array}$

$-0.026904$

$-0.940892$

$-1.033376$

2. 335638

3.177290

1.710387

1.986978

0.593863

$-0.102627$

0.560003

$-0.166223$

1.653298

1.882817

1. 108188

0.233019

1.964863

1.595577

3. 255645

4. 114951

3. 303680

4. 204109

2. 042019

1.740358

$-1.880161$

$-1.863141$

$-3.292923$

$-1.445546$

$-2.663356$

$-1.602133$

$-3.233616$

$-2.123043$

$-2.552446$

$-0.860305$

$-2.167850$

$-4.015195$

$-3.614732$

$-3.305470$

$-2.134703$

$-1.436194$

- 0.439995

$-3.493487$

$-3.020611$

$-1.877869$

$-2.405131$

$-0.795719$

$-1.212188$

$-4.064031$

$-2.849499$

$-3.616014$
$-1.146961$

$-1.449655$

1.142694

2.248550

2.769956

1.681050

$-3.719089$

$-3.700874$

$-3.917471$

$-1.415181$

$-1.419938$

$-0.062167$

$-1.718498$

$-0.368172$

$-1.950708$

1.939598

$\odot .771463$

$\odot .320675$

3. 053324

1. 459631

2. 651473

3. 606680

3. 152835

2. 370682

0.361952

0.940604

$-0.747266$

1.138504

0.586108

2. 250739

2. 680075

2. 614712

3.416493

1.750513

1.794288

$\odot .861182$

0.056510

$-1.213374$

$-1.720818$

$-2.056991$

$-2.653514$

1.964170

$-2.460977$

$-1.090546$

$-0.842422$

$-0.654065$

0.010608

1. 347045

0.274540

1. 455297

2. 712088

2.449513

$-2.408628$

$-0.597580$

$-1.544884$

$\odot .553058$

0.172705

$-0.697878$

1.772787

$\odot .490068$

2. 187529

3. 038352

3. 474350

2. 645586

$-3.064263$

$-1.846295$

$-3.116871$

$-3.031213$

3.065892

$-1.774334$

$-1.176754$

0.065959

๑. . 018011
$-2.760227$

$-3.077403$

0.115798

1.915116

$-0.397848$

0.505742

$-1.734806$

$-3.370461$

$-1.959167$

$-3.803760$

$-2.159479$

$-2.703274$

$-4.129485$

$-3.017057$

$-2.712074$

$-0.164957$

$-1.128297$

0.486102

1.866757

. 571407

2.353560

$-0.504836$

0.032083

$-1.392827$

$-0.628695$

0.744757

$-1.534434$

$-1.264255$

$-0.873239$

$-0.640464$

0.312417

$-1.425894$

$-1.220758$

$-2.574558$

$-3.374843$

$-2.480520$

$-3.165133$

$\odot .504326$

$\odot .921913$

$-0.360089$

$-1.185648$

0.068593

$-0.369741$

1. 232991

1.785295

1.508438

2. 326082

1. 763721

2. 874736

1.153975

2. 339158

$-0.924706$

$-3.221045$

$-2.553676$

$-2.052301$

3. 679142

3. 299180

2. 477191

1. 914098

0.753478

๑. 340798

3. 125944

1.553941

2.764581

$-1.289618$

$-0.085322$

$-0.557646$

$-3.631183$

$-2.882581$

$-4.022711$

$-2.971918$

$-3.334290$

$-1.735089$
TS2

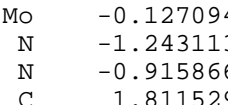

$\odot .540219$

1.337313

$-0.853039$

2. 210804
$-0.270854$

0.819930

$-0.956592$

$-0.657301$ 


\begin{tabular}{|c|c|c|c|}
\hline $\mathrm{H}$ & 2.384512 & 2.580220 & ๑. 182034 \\
\hline C & ๑. 621961 & 2.763879 & -1.151492 \\
\hline $\mathrm{H}$ & ๑. 092805 & 3.615368 & -0.747889 \\
\hline C & ๑. 159224 & 1.923832 & -2.216390 \\
\hline $\mathrm{H}$ & $-\odot .720514$ & 2.096257 & -2.822603 \\
\hline C & 1.157864 & $\odot .923121$ & -2.447800 \\
\hline $\mathrm{H}$ & 1.101606 & ๑. 135118 & -3.185601 \\
\hline C & 2.144732 & 1.067081 & -1.465704 \\
\hline $\mathrm{H}$ & 2.992533 & 0.416053 & -1.309350 \\
\hline C & 1.107170 & -0.505168 & 1.365489 \\
\hline $\mathrm{H}$ & ๑. 617057 & -0.037939 & 2.223225 \\
\hline C & $\odot .935250$ & -1.978591 & 1.265016 \\
\hline $\mathrm{H}$ & -0.023976 & -2.476543 & 1.322504 \\
\hline C & 2.151550 & -2.564896 & 1.098197 \\
\hline $\mathrm{H}$ & 2.342738 & -3.625225 & ๑. 971736 \\
\hline C & 3.177187 & -1.531922 & 1.140478 \\
\hline $\mathrm{H}$ & 4.244380 & -1.713021 & 1.063656 \\
\hline C & 2.578493 & -0.323026 & 1.322971 \\
\hline $\mathrm{H}$ & 3.081006 & ๑. 627608 & 1.452590 \\
\hline C & -2.433035 & 1.721207 & 1.545327 \\
\hline C & -2.066673 & 1.757727 & 3.045529 \\
\hline C & -3.575387 & $\odot .715726$ & 1.305148 \\
\hline C & -2.855203 & 3.129657 & 1.074364 \\
\hline C & -2.607296 & -2.590146 & $-\odot .615221$ \\
\hline C & $-\odot .634615$ & -2.941722 & -2.161401 \\
\hline C & -2.453751 & -1.289790 & -2.775891 \\
\hline C & -1.647991 & -1.912332 & -1.615561 \\
\hline $\mathrm{H}$ & -2.932416 & 2.070664 & 3.639046 \\
\hline $\mathrm{H}$ & -1.248071 & 2.460573 & 3.224832 \\
\hline $\mathrm{H}$ & -1.753691 & $\odot .767789$ & 3.390738 \\
\hline $\mathrm{H}$ & -4.473387 & 1.010750 & 1.859199 \\
\hline $\mathrm{H}$ & -3.279913 & $-\odot .284486$ & 1.633957 \\
\hline $\mathrm{H}$ & -3.828051 & ๑. 667690 & ๑. 241693 \\
\hline $\mathrm{H}$ & -3.742840 & 3.464114 & 1.622167 \\
\hline $\mathrm{H}$ & -3.093055 & 3.123633 & $\odot .0 \odot 6075$ \\
\hline $\mathrm{H}$ & -2.050456 & 3.850556 & 1.245827 \\
\hline $\mathrm{H}$ & -3.162202 & -3.395452 & -1.108912 \\
\hline $\mathrm{H}$ & -3.325051 & -1.868682 & -0.217564 \\
\hline $\mathrm{H}$ & -2.052132 & -3.021830 & ○. 222145 \\
\hline $\mathrm{H}$ & -1.157182 & -3.779033 & -2.636954 \\
\hline $\mathrm{H}$ & -0.011558 & -3.326939 & -1.350385 \\
\hline $\mathrm{H}$ & ๑. 022602 & -2.477450 & -2.902583 \\
\hline $\mathrm{H}$ & -1.786922 & -0.799054 & -3.490917 \\
\hline $\mathrm{H}$ & -3.164238 & $-\odot .547682$ & -2.399786 \\
\hline $\mathrm{H}$ & -3.012719 & -2.068789 & -3.305322 \\
\hline
\end{tabular}

\section{TS3}

$\begin{array}{rrrr}\text { Mo } & -\odot .667352 & -0.640182 & 0.804474 \\ \mathrm{~N} & -0.501370 & -1.520648 & -0.696039 \\ \mathrm{~N} & \odot .381537 & 0.747901 & 0.753494 \\ \mathrm{C} & -3.143629 & -1.149866 & 0.881391 \\ \mathrm{H} & -3.502018 & -1.770467 & 0.072321 \\ \mathrm{C} & -2.892863 & 0.258946 & 0.804213 \\ \mathrm{H} & -3.147726 & 0.903390 & -0.027006 \\ \mathrm{C} & -2.436618 & 0.690736 & 2.094392 \\ \mathrm{H} & -2.168823 & 1.703699 & 2.360410 \\ \mathrm{C} & -2.282772 & -0.444686 & 2.895862 \\ \mathrm{H} & -1.893093 & -0.465236 & 3.905233 \\ \mathrm{C} & -2.715832 & -1.591379 & 2.139614 \\ \mathrm{H} & -2.715043 & -2.615552 & 2.489096 \\ \mathrm{C} & \odot .561364 & -1.989719 & 2.172616 \\ \mathrm{H} & -0.163575 & -2.208139 & 2.961056 \\ \mathrm{C} & 1.060052 & -3.188616 & 1.449171 \\ \mathrm{H} & 0.414916 & -3.939184 & 1.009696 \\ \mathrm{C} & 2.420435 & -3.175371 & 1.445481 \\ \mathrm{H} & 3.068262 & -3.909408 & 0.977824 \\ \mathrm{C} & 2.882558 & -2.013043 & 2.192877 \\ \mathrm{H} & 3.923770 & -1.758179 & 2.360940 \\ \mathrm{C} & 1.802046 & -1.322335 & 2.646729 \\ \mathrm{H} & 1.812601 & -0.431272 & 3.262745 \\ \mathrm{C} & -0.116731 & -1.870292 & -2.047954 \\ \mathrm{C} & -0.087538 & -3.409900 & -2.158480 \\ \mathrm{C} & 1.273094 & -1.290860 & -2.374693 \\ \mathrm{C} & -1.177812 & -1.294530 & -3.010144 \\ \mathrm{C} & 2.637270 & 1.469203 & 0.182289 \\ \mathrm{C} & 1.207670 & 2.782324 & 1.802905 \\ \mathrm{C} & 0.595487 & 2.718817 & -0.649405 \\ \mathrm{C} & 1.200949 & 1.918388 & 0.522992 \\ \mathrm{H} & 0.649758 & -3.825580 & -1.467405 \\ \mathrm{H} & \odot .178501 & -3.712479 & -3.177121 \\ \mathrm{H} & -1.067213 & -3.831643 & -1.914600 \\ \mathrm{H} & 1.582922 & -1.580999 & -3.384972 \\ \mathrm{H} & 2.015126 & -1.659597 & -1.661292 \\ \mathrm{H} & 1.255277 & -0.198851 & -2.321737\end{array}$

$\begin{array}{rrrr}\mathrm{H} & -0.934674 & -1.564251 & -4.043575 \\ \mathrm{H} & -1.214294 & -0.203404 & -2.937104 \\ \mathrm{H} & -2.170262 & -1.690482 & -2.775035 \\ \mathrm{H} & 3.286113 & 2.344542 & 0.067323 \\ \mathrm{H} & 2.654766 & 0.898960 & -0.749546 \\ \mathrm{H} & 3.035830 & 0.832315 & 0.975286 \\ \mathrm{H} & 1.817214 & 3.680665 & 1.656330 \\ \mathrm{H} & 1.621304 & 2.218240 & 2.643611 \\ \mathrm{H} & 0.191938 & 3.094795 & 2.064199 \\ \mathrm{H} & 1.195202 & 3.614472 & -0.844135 \\ \mathrm{H} & -0.426890 & 3.033121 & -0.417657 \\ \mathrm{H} & 0.571765 & 2.113036 & -1.559566\end{array}$

\section{TS4}

Mo

0.149367

$-0.562614$

$-1.129402$

2. 374906

3. 210501

1.335830

1. 208716

0.379992

$-0.520906$

0.940320

$\odot .461442$

2.130529

2.748404

1.194201

0.271679

2. 386535

2. 459391

3. 454452

4.494855

2. 955297

3.536238

1.554088

0.939619

$-1.569435$

$-2.007131$

$-2.776756$

$-0.963256$

$-3.169538$

$-1.728532$

$-3.029657$

$-2.253948$

$-2.775619$

$-1.158974$

$-2.413673$

$-3.553062$

$-3.204266$

$-2.472784$

$-1.698543$

$-0.641122$

$-0.092949$

$-4.018913$

$-3.557918$

$-2.619466$

$-2.562234$

$-1.151806$

$-1.079037$

$-3.890386$

$-2.388399$

$-3.391650$

0.088784

$\odot .842809$

$-0.822822$

1.295315

1. 218555

2. 231008

3. 002306

1.885623

2. 436576

0.813498

$\odot .329377$

0.417859

$-0.423783$

$-2.200363$

$-2.761091$

$-2.516470$

$-3.162423$

$-1.861132$

$-1.908248$

$-1.116431$

$-0.503218$

$-1.305677$

$-1.118074$

1. 271478

0.049379

1. 848739

2. 351792

$-2.090837$

$-2.558596$

$-0.394902$

$-1.463232$

0.340783

$-0.366016$

$-0.733188$

2.176164

1. 095707

2. 709775

2. 689176

3. 218957

1. 956691

$-2.598014$

$-1.323195$

$-2.825709$

$-3.063963$

$-3.306477$

$-2.122904$

$-0.850099$

0.058339

0.397068

$-0.525517$

0.886597

$-1.284270$

$-1.102766$

$-0.423611$

$-1.044153$

$-0.298059$

$-2.057836$

$-2.294137$

$-2.833878$

$-3.673306$

$-2.218858$

$-2.493132$

0.002227

$-0.054784$

$-0.687764$

$-1.555049$

$-0.046477$

$-0.351384$

1.035354

1.714483

1.096376

1.965407

1.835023

2. 671023

1.066972

2.755953

$-0.852457$

$-2.879127$

$-2.725718$

$-1.925185$

3. 395319

3. 223359

2. 024893

1.767072

0.399741

0.463616

3. 494973

2. 171273

3.287894

$-1.323193$

$-0.177241$

$-0.257009$

$-3.378782$

$-2.327880$

$-3.643822$

$-3.227279$

$-3.487260$

$-2.063926$

\section{TS5}

$\begin{array}{rr}\text { Mo } & 0.215887 \\ \mathrm{~N} & -0.259027 \\ \mathrm{~N} & -1.185954 \\ \mathrm{C} & 2.172576 \\ \mathrm{H} & 2.305612 \\ \mathrm{C} & 0.874219 \\ \mathrm{H} & 0.045971 \\ \mathrm{C} & 1.192376 \\ \mathrm{H} & 0.456316 \\ \mathrm{C} & 2.550688 \\ \mathrm{H} & 3.092553 \\ \mathrm{C} & 3.158247 \\ \mathrm{H} & 4.221362 \\ \mathrm{C} & 1.132350 \\ \mathrm{H} & 0.304647 \\ \mathrm{C} & 1.683783 \\ \mathrm{H} & 1.104001 \\ \mathrm{C} & 3.031467\end{array}$

$\begin{array}{rr}-0.139009 & -0.540183 \\ 0.784567 & 0.855050 \\ -0.803975 & -1.323250 \\ 1.809761 & -1.352513 \\ 2.402144 & -0.455889 \\ 1.465032 & -1.946072 \\ 2.174090 & -1.924908 \\ 0.723463 & -3.173183 \\ 0.350635 & -3.874272 \\ 0.554167 & -3.237734 \\ 0.001464 & -3.996835 \\ 1.225168 & -2.107261 \\ 1.246588 & -1.895918 \\ -1.963286 & 0.360975 \\ -2.420522 & 0.903807 \\ -2.594387 & -0.844216 \\ -3.140434 & -1.577904 \\ -2.338504 & -0.884744\end{array}$




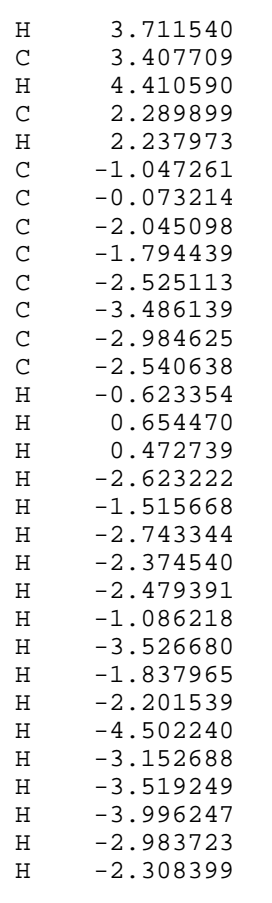

$\begin{array}{rr}-2.621780 & -1.680046 \\ -1.609060 & 0.308173 \\ -1.266575 & 0.536644 \\ -1.413867 & 1.077463 \\ -0.906809 & 2.032774 \\ 1.427608 & 1.890152 \\ 1.917755 & 2.983641 \\ 0.417697 & 2.490916 \\ 2.628419 & 1.274796 \\ -1.682939 & -3.134697 \\ 0.021328 & -1.537319 \\ -2.328920 & -0.733483 \\ -1.188027 & -1.672156 \\ 2.424783 & 3.783686 \\ 2.618387 & 2.564436 \\ 1.075767 & 3.418838 \\ 0.887826 & 3.293653 \\ -0.444347 & 2.907423 \\ 0.058927 & 1.730010 \\ 3.149108 & 2.044175 \\ 2.298916 & 0.488759 \\ 3.338561 & 0.838090 \\ -2.006823 & -3.437681 \\ -2.526238 & -3.247585 \\ -0.883933 & -3.808079 \\ -0.257229 & -1.836698 \\ 0.844213 & -2.176566 \\ 0.377217 & -0.504330 \\ -2.660432 & -0.991033 \\ -1.995788 & 0.308210 \\ -3.184210 & -0.821415 \\ & \end{array}$

TS6

\begin{tabular}{|c|c|c|c|}
\hline Mo & $\odot .513472$ & 1.020788 & $-\odot .431903$ \\
\hline $\mathrm{N}$ & $-\odot .954103$ & 1.551945 & ๑. 351527 \\
\hline $\mathrm{N}$ & ๑. 407066 & $-\odot .696425$ & $-\odot .649475$ \\
\hline C & 1.625659 & 3. 224771 & -1.657199 \\
\hline $\mathrm{H}$ & 2.058699 & 4.045465 & -1.099376 \\
\hline C & 0.276240 & 3.073029 & -1.980577 \\
\hline $\mathrm{H}$ & -0.528280 & 3.739038 & -1.702380 \\
\hline C & 0.119244 & 1.804318 & -2.635054 \\
\hline $\mathrm{H}$ & $-\odot .791554$ & 1.433862 & -3.086724 \\
\hline C & 1.436075 & 1. 255046 & -2.824905 \\
\hline $\mathrm{H}$ & 1.660368 & ๑. 310981 & -3.301474 \\
\hline C & 2.347249 & 2. 098004 & -2.190234 \\
\hline $\mathrm{H}$ & 3.409537 & 1.923001 & -2.086689 \\
\hline C & 1.604069 & 1.153123 & 1.831436 \\
\hline $\mathrm{H}$ & 1.059626 & 1.995800 & 2.237295 \\
\hline C & 1.710449 & -0.124983 & 2.435386 \\
\hline $\mathrm{H}$ & 1.046943 & -0.515236 & 3.198110 \\
\hline C & 2.808500 & -0.792741 & 1.867255 \\
\hline $\mathrm{H}$ & 3.128740 & -1.799534 & 2.113826 \\
\hline C & 3.397367 & ๑ . 034701 & ๑.895991 \\
\hline $\mathrm{H}$ & 4.252056 & -0.212554 & $\odot .277386$ \\
\hline C & 2.679002 & 1.255815 & ๑.857777 \\
\hline $\mathrm{H}$ & 3.061704 & 2.189292 & ๑. 469468 \\
\hline C & -2.260933 & 1.643332 & $\odot .963792$ \\
\hline C & -2.165462 & 1.106459 & 2.407786 \\
\hline C & -3.272801 & $\odot .809635$ & 0.150325 \\
\hline C & -2.695781 & 3.125272 & $\odot .978775$ \\
\hline C & -0.645178 & -2.518283 & 0.595736 \\
\hline C & 1.257846 & -2.931351 & -1.019124 \\
\hline C & -1.008993 & -2.225341 & -1.896302 \\
\hline C & -0.001424 & -2.083004 & -0.736569 \\
\hline $\mathrm{H}$ & -3.147501 & 1.149623 & 2.891187 \\
\hline $\mathrm{H}$ & -1.465677 & 1.704518 & 2.998121 \\
\hline $\mathrm{H}$ & -1.816348 & ๑.๑7०687 & 2.411319 \\
\hline $\mathrm{H}$ & -4.275999 & 0.902310 & 0.580540 \\
\hline $\mathrm{H}$ & -2.994535 & -0.247365 & 0.150570 \\
\hline $\mathrm{H}$ & -3.309536 & 1.155808 & -0.887151 \\
\hline $\mathrm{H}$ & -3.674984 & 3.235080 & 1.457573 \\
\hline $\mathrm{H}$ & -2.768211 & 3.516685 & $-\odot .040680$ \\
\hline $\mathrm{H}$ & -1.970844 & 3.731973 & 1.529112 \\
\hline $\mathrm{H}$ & -0.921796 & -3.577436 & $\odot .553138$ \\
\hline $\mathrm{H}$ & -1.547165 & -1.935212 & $\odot .800673$ \\
\hline $\mathrm{H}$ & ๑. 054826 & -2.370021 & 1.421369 \\
\hline $\mathrm{H}$ & ๑. 995879 & -3.993537 & -1.076486 \\
\hline $\mathrm{H}$ & 1.994877 & -2.791429 & -0.224898 \\
\hline $\mathrm{H}$ & 1.714996 & -2.634310 & -1.967590 \\
\hline $\mathrm{H}$ & -1.312962 & -3.272603 & -1.998530 \\
\hline $\mathrm{H}$ & -0.560444 & -1.904401 & -2.841205 \\
\hline $\mathrm{H}$ & -1.902277 & -1.621192 & -1.714349 \\
\hline
\end{tabular}

\section{B3LYP/b1; Mo( $\left.\left.\eta^{5}-C p\right)\left(\eta^{1}-C p\right)(N M e)_{2}\right]$}

Mo

$\begin{array}{rr}\text { Mo } & -0.471046 \\ \mathrm{~N} & -1.231185 \\ \mathrm{~N} & -1.655521 \\ \mathrm{C} & 1.867645 \\ \mathrm{H} & 2.710508 \\ \mathrm{C} & 1.019127 \\ \mathrm{H} & 1.082292 \\ \mathrm{C} & -0.027157 \\ \mathrm{H} & -0.831344 \\ \mathrm{C} & 0.267111 \\ \mathrm{H} & -0.339505 \\ \mathrm{C} & 1.414560 \\ \mathrm{H} & 1.860793 \\ \mathrm{C} & 0.644328 \\ \mathrm{H} & -0.230632 \\ \mathrm{C} & 1.413325 \\ \mathrm{H} & 0.972243 \\ \mathrm{C} & 2.732679 \\ \mathrm{H} & 3.545571 \\ \mathrm{C} & 2.884516 \\ \mathrm{H} & 3.826667 \\ \mathrm{C} & 1.657938 \\ \mathrm{H} & 1.430330 \\ \mathrm{C} & -2.332799 \\ \mathrm{H} & -2.044920 \\ \mathrm{H} & -3.216287 \\ \mathrm{H} & -2.613597 \\ \mathrm{H} & -2.609019 \\ \mathrm{H} & -2.629401 \\ \mathrm{H} & -3.689445 \\ \mathrm{C} & -2.695476 \\ & \end{array}$

Bo

Mo

$\mathrm{N}$
$\mathrm{N}$

$-0.303077$

0.616807

$-2.919283$

$-3.177045$

$-2.925562$

$-3.260738$

$-2.478236$

$-2.391898$

$-2.176349$

$-1.770578$

$-2.414897$

$-2.253660$

0.657318

0.046674

2. 019157

2. 238664

2. 911733

3. 980785

2. 198677

2. 658462

$\odot .874799$

$\odot .095969$

$\odot .310789$

$\odot .752190$

1.102975

$-0.436818$

2. 528595

1. 368215

1. 970247

1.663697

TS1

$\begin{array}{rr}\text { Mo } & -0.469109 \\ \mathrm{~N} & -1.305894 \\ \mathrm{~N} & -1.588902 \\ \mathrm{C} & 1.864046 \\ \mathrm{H} & 2.714530 \\ \mathrm{C} & 1.295772 \\ \mathrm{H} & 1.641203 \\ \mathrm{C} & 0.145298 \\ \mathrm{H} & -0.516152 \\ \mathrm{C} & 0.031153 \\ \mathrm{H} & -0.743242 \\ \mathrm{C} & 1.109536\end{array}$

$\odot .267357$

0.967774

$-0.773272$

1.130339

0.834737

2. 229362

2. 921941

2. 181835

2. 898477

1.107263

0.821446

0.452072

$-0.439982$

$-1.351722$

1.810460

$-2.233924$

$-2.828738$

$-2.175504$

$-2.700363$

$-1.296580$

$-1.074171$

$-0.817059$

$-0.157311$

1.165371

0.871537

0.579043

2. 227047

$-2.579454$

$-1.459691$

$-1.169958$

$-1.521110$

0.081836

$-0.635372$

1.374110

$-0.801053$

$-1.708468$

0.507474

$\odot .787581$

1. 411891

2. 485333

0.654365

1.037054

$-0.716602$

$-1.551255$

$-1.427825$

$-2.326844$

$-1.467509$

$-1.810111$

$-0.996691$

$-0.882274$

$-0.679288$

$-0.291428$

$-0.956579$

$-0.856040$

$-0.726278$

$-1.724222$

0.023862

$-0.599458$

1. 880339

3. 229816

2. 641734

2. 336401

0.249970

0.965892

$-0.854537$

0.843946

0.299879

2. 010287

2. 505001

2. 350785

3. 186937

1. 408920

1.410218

0.502577
$-0.110991$

1.305258

$-0.849263$

$-0.755209$

$-0.148020$

$-0.521655$

0.306088

$-1.490576$

$-1.596254$

$-2.399516$

-3. 248291

$-1.936155$

$-2.351499$

0.977941

1.449569

0.070657

$-0.720476$

0.410093

$-0.080794$

1.560005

2. 050233

1. 914708

2. 743291

2. 193231

3. 212065

1.899133

2. 230079

$-1.192498$

$-2.569772$

$-1.164851$

$-1.474130$

-0.257469
-1.842445
-0.113734
0.054966
-0.473705
-0.488783
-1.478375
0.534980
0.433273
1.683835
2.609977
1.375223
2.044758
0.904741
0.773077
0.321227
-0.682475
1.238109
1.092329
2.464462
3.367455
2.292262
3.038132
-3.129358
-3.258031
-3.269713
-3.924732
0.423917
0.487587
-1.088030
-0.078659

$-0.129973$

1.238061 $-0.877200$

$-1.024629$

$-0.641040$

$-0.443534$

0.453499

$-1.193591$

$-1.011383$

$-2.273053$

- .028689

$-2.178076$ 


$\begin{array}{lr}\mathrm{H} & 1.279125 \\ \mathrm{C} & 0.657615 \\ \mathrm{H} & -0.212538 \\ \mathrm{C} & 1.436215 \\ \mathrm{H} & 1.002851 \\ \mathrm{C} & 2.752109 \\ \mathrm{H} & 3.569520 \\ \mathrm{C} & 2.893718 \\ \mathrm{H} & 3.831789 \\ \mathrm{C} & 1.663518 \\ \mathrm{H} & 1.427617 \\ \mathrm{C} & -2.458365 \\ \mathrm{H} & -2.205052 \\ \mathrm{H} & -3.297061 \\ \mathrm{H} & -2.793076 \\ \mathrm{H} & -2.433808 \\ \mathrm{H} & -2.439870 \\ \mathrm{H} & -3.582253 \\ \mathrm{C} & -2.557994\end{array}$

TS2

$\begin{array}{rrrr}\text { Mo } & -0.367096 & 0.420931 & -0.002309 \\ \mathrm{~N} & -1.354792 & 1.019784 & 1.317981 \\ \mathrm{~N} & -1.319298 & -0.769321 & -0.830936 \\ \mathrm{C} & 1.651346 & 2.016166 & -0.283203 \\ \mathrm{H} & 2.334469 & 2.183007 & 0.538261 \\ \mathrm{C} & 0.461401 & 2.720162 & -0.521915 \\ \mathrm{H} & 0.042034 & 3.500808 & 0.097195 \\ \mathrm{C} & -0.165987 & 2.138123 & -1.670146 \\ \mathrm{H} & -1.087290 & 2.475605 & -2.127224 \\ \mathrm{C} & 0.728187 & 1.152073 & -2.203667 \\ \mathrm{H} & 0.538892 & 0.533433 & -3.070226 \\ \mathrm{C} & 1.818633 & 1.046727 & -1.333433 \\ \mathrm{H} & 2.627941 & 0.334545 & -1.403541 \\ \mathrm{C} & 0.912189 & -1.003423 & 1.264660 \\ \mathrm{H} & 0.544649 & -0.696422 & 2.247122 \\ \mathrm{C} & 0.598340 & -2.401161 & 0.868097 \\ \mathrm{H} & -0.391724 & -2.836578 & 0.926491 \\ \mathrm{C} & 1.733686 & -3.010316 & 0.431351 \\ \mathrm{H} & 1.814326 & -4.022905 & 0.050596 \\ \mathrm{C} & 2.847112 & -2.082937 & 0.576485 \\ \mathrm{H} & 3.879748 & -2.309876 & 0.332088 \\ \mathrm{C} & 2.381578 & -0.910803 & 1.089342 \\ \mathrm{H} & 2.975454 & -0.045874 & 1.358597 \\ \mathrm{C} & -2.573141 & 1.051377 & 2.064370 \\ \mathrm{H} & -2.366006 & 0.810192 & 3.116324 \\ \mathrm{H} & -3.317472 & 0.335965 & 1.684142 \\ \mathrm{H} & -3.012200 & 2.058552 & 2.045038 \\ \mathrm{H} & -1.777409 & -2.693951 & -1.415308 \\ \mathrm{H} & -2.107604 & -1.451473 & -2.638741 \\ \mathrm{H} & -3.192152 & -1.626220 & -1.232563 \\ \mathrm{C} & -2.145515 & -1.669888 & -1.562527\end{array}$

TS3

\begin{tabular}{rrrr} 
Mo & -0.559023 & 0.300990 & -0.025958 \\
N & 0.051296 & -0.040615 & -1.619513 \\
N & 0.372998 & 1.634022 & 0.590735 \\
C & -2.730532 & -1.119907 & 0.475429 \\
H & -2.694423 & -2.201207 & 0.500956 \\
C & -2.945512 & -0.328371 & -0.658462 \\
H & -3.075117 & -0.681062 & -1.672331 \\
C & -2.828218 & 1.045048 & -0.261551 \\
H & -2.984126 & 1.904963 & -0.900225 \\
C & -2.677015 & 1.074467 & 1.164750 \\
H & -2.568629 & 1.965759 & 1.767281 \\
C & -2.564042 & -0.247258 & 1.609300 \\
H & -2.380852 & -0.564376 & 2.627674 \\
C & 0.547298 & -1.364171 & 1.074780 \\
H & -0.266675 & -1.879789 & 1.589825 \\
C & 1.316303 & -2.191725 & 0.110718 \\
H & 0.862393 & -2.806755 & -0.657255 \\
C & 2.646360 & -2.039471 & 0.353859 \\
H & 3.456639 & -2.500411 & -0.201426 \\
C & 2.818193 & -1.161253 & 1.504343 \\
H & 3.774752 & -0.874235 & 1.928541 \\
C & 1.591843 & -0.783070 & 1.956373 \\
H & 1.379733 & -0.155786 & 2.814015 \\
C & 0.862968 & -0.016680 & -2.791794 \\
H & 1.642070 & -0.784319 & -2.688704 \\
H & 1.356970 & 0.955204 & -2.926450 \\
H & 0.274722 & -0.242012 & -3.691493 \\
H & 2.221247 & 2.225621 & 1.270767 \\
H & 0.980907 & 3.502242 & 1.315082 \\
\hline
\end{tabular}

$\begin{array}{rr}-0.356476 & -2.812481 \\ -1.287199 & 1.054517 \\ -1.721408 & 1.557580 \\ -2.231078 & 0.220111 \\ -2.882812 & -0.529387 \\ -2.146194 & 0.566525 \\ -2.706373 & 0.124531 \\ -1.178846 & 1.645593 \\ -0.915654 & 2.123124 \\ -0.678159 & 1.954148 \\ 0.040255 & 2.730006 \\ 1.138385 & 2.064443 \\ 0.898144 & 3.106375 \\ 0.496798 & 1.754256 \\ 2.185024 & 2.047882 \\ -2.701464 & -1.279004 \\ -1.573054 & -2.649461 \\ -1.347003 & -1.297896 \\ -1.646462 & -1.558853\end{array}$

1. 714411

1. 361963

3.017461

2. 651195

$-0.239120$

TS4

$\begin{array}{rrrr}\text { Mo } & -0.310911 & 0.366148 & -0.022930 \\ \mathrm{~N} & -\odot .921534 & 1.206731 & 1.399660 \\ \mathrm{~N} & -1.705961 & -0.468064 & -0.643145 \\ \mathrm{C} & 1.889365 & 1.405539 & -0.814175 \\ \mathrm{H} & 2.758644 & 1.340476 & -0.177410 \\ \mathrm{C} & 0.887867 & 2.3890 \odot 5 & -0.760089 \\ \mathrm{H} & 0.831798 & 3.210242 & -0.059692 \\ \mathrm{C} & -0.121188 & 2.033513 & -1.712195 \\ \mathrm{H} & -1.014813 & 2.603315 & -1.931103 \\ \mathrm{C} & 0.353562 & 0.891930 & -2.443946 \\ \mathrm{H} & -0.185499 & 0.382181 & -3.231105 \\ \mathrm{C} & 1.561489 & 0.485533 & -1.869064 \\ \mathrm{H} & 2.127658 & -0.398826 & -2.119732 \\ \mathrm{C} & 0.550372 & -1.970522 & 0.557418 \\ \mathrm{H} & -0.420211 & -2.443633 & 0.627071 \\ \mathrm{C} & 1.632406 & -2.435438 & -0.223897 \\ \mathrm{H} & 1.558508 & -3.137417 & -1.046402 \\ \mathrm{C} & 2.814434 & -1.841000 & 0.258937 \\ \mathrm{H} & 3.808506 & -1.998964 & -0.146347 \\ \mathrm{C} & 2.496028 & -0.988812 & 1.327740 \\ \mathrm{H} & 3.193610 & -0.388770 & 1.900334 \\ \mathrm{C} & 1.095847 & -1.043791 & 1.541715 \\ \mathrm{H} & \odot .597442 & -0.748504 & 2.454975 \\ \mathrm{C} & -2.087913 & 1.459364 & 2.187207 \\ \mathrm{H} & -1.899119 & 1.171692 & 3.231417 \\ \mathrm{H} & -2.966095 & 0.903145 & 1.823465 \\ \mathrm{H} & -2.324807 & 2.532652 & 2.189928 \\ \mathrm{H} & -2.675082 & -2.187602 & -1.270717 \\ \mathrm{H} & -2.640360 & -\odot .875406 & -2.467032 \\ \mathrm{H} & -3.734391 & -0.775127 & -1.061586 \\ \mathrm{C} & -2.738450 & -1.096888 & -1.394199\end{array}$

\section{TS5}

$\begin{array}{rrrr}\text { Mo } & -0.383418 & 0.113932 & 0.260896 \\ \mathrm{~N} & -0.939167 & 0.962143 & 1.669157 \\ \mathrm{~N} & -1.793489 & -0.525116 & -0.522675 \\ \mathrm{C} & 1.472243 & 2.188119 & -0.446340 \\ \mathrm{H} & 1.547422 & 2.785713 & 0.453666 \\ \mathrm{C} & 0.213972 & 1.794085 & -1.088296 \\ \mathrm{H} & -0.653525 & 2.454045 & -1.082909 \\ \mathrm{C} & 0.602859 & 1.062086 & -2.297843 \\ \mathrm{H} & -0.091435 & 0.660488 & -3.025311 \\ \mathrm{C} & 1.970050 & 0.942579 & -2.308981 \\ \mathrm{H} & 2.559343 & 0.404063 & -3.042337 \\ \mathrm{C} & 2.508639 & 1.639054 & -1.161174 \\ \mathrm{H} & 3.560868 & 1.698290 & -0.907556 \\ \mathrm{C} & 0.536173 & -1.731075 & 1.125285 \\ \mathrm{H} & -0.289810 & -2.213369 & 1.647912 \\ \mathrm{C} & 1.105890 & -2.310902 & -0.095278 \\ \mathrm{H} & 0.542459 & -2.851110 & -0.845979 \\ \mathrm{C} & 2.446828 & -2.014648 & -0.125091 \\ \mathrm{H} & 3.135389 & -2.253101 & -0.927634 \\ \mathrm{C} & 2.798570 & -1.307415 & 1.086747 \\ \mathrm{H} & 3.789439 & -0.938767 & 1.326209 \\ \mathrm{C} & 1.673086 & -1.167052 & 1.859631 \\ \mathrm{H} & 1.604886 & -0.689679 & 2.829209 \\ \mathrm{C} & -1.867001 & 1.484791 & 2.625705 \\ \mathrm{H} & -1.625309 & 1.110812 & 3.629064 \\ \mathrm{H} & -2.901885 & 1.203991 & 2.390077 \\ \mathrm{H} & -1.802401 & 2.580335 & 2.651483 \\ \mathrm{H} & -3.339051 & -1.888469 & -0.807669 \\ \mathrm{H} & -3.509225 & -0.359044 & -1.689862 \\ \mathrm{H} & -3.811511 & -0.409817 & 0.065127 \\ \mathrm{C} & -3.180291 & -0.804266 & -0.741935\end{array}$

TS6

$\begin{array}{rrrr}\text { Mo } & 0.032615 & 0.497782 & 0.062471 \\ \mathrm{~N} & 1.451264 & 0.780392 & 1.042356 \\ \mathrm{~N} & -1.308934 & 0.500427 & 1.150587 \\ \mathrm{C} & 0.683002 & 1.043137 & -2.567066 \\ \mathrm{H} & 1.362183 & 0.429213 & -3.145081 \\ \mathrm{C} & 1.045331 & 2.084335 & -1.708680 \\ \mathrm{H} & 2.051724 & 2.405608 & -1.478535 \\ \mathrm{C} & -0.147387 & 2.568920 & -1.075477 \\ \mathrm{H} & -0.212192 & 3.430479 & -0.423553 \\ \mathrm{C} & -1.261603 & 1.881546 & -1.676233 \\ \mathrm{H} & -2.302720 & 2.034313 & -1.426516 \\ \mathrm{C} & -0.751069 & 0.925527 & -2.554689\end{array}$




$\begin{array}{rrrr}\mathrm{H} & -1.329277 & 0.197042 & -3.106721 \\ \mathrm{C} & 0.579470 & -1.923774 & 0.248147 \\ \mathrm{H} & 1.661344 & -1.879793 & 0.271073 \\ \mathrm{C} & -0.237978 & -2.533438 & 1.237382 \\ \mathrm{H} & 0.065280 & -2.748792 & 2.255406 \\ \mathrm{C} & -1.499798 & -2.782261 & 0.676735 \\ \mathrm{H} & -2.344727 & -3.222535 & 1.195794 \\ \mathrm{C} & -1.506450 & -2.332088 & -0.657523 \\ \mathrm{H} & -2.345675 & -2.373424 & -1.341793 \\ \mathrm{C} & -0.226689 & -1.807775 & -0.956549 \\ \mathrm{H} & \odot .175380 & -1.663724 & -1.949991 \\ \mathrm{C} & 2.196298 & 0.939606 & 2.247640 \\ \mathrm{H} & 2.724002 & 0.002758 & 2.479301 \\ \mathrm{H} & 1.552445 & 1.188799 & 3.102757 \\ \mathrm{H} & 2.954310 & 1.727751 & 2.139640 \\ \mathrm{H} & -2.417033 & -0.627470 & 2.442426 \\ \mathrm{H} & -3.097638 & 0.984345 & 2.109471 \\ \mathrm{H} & -1.683317 & 0.817864 & 3.190996 \\ \mathrm{C} & -2.165262 & 0.431531 & 2.283226\end{array}$

